\title{
RNA-seq reveals the pan-transcriptomic impact of attenuating the gliotoxin self-protection mechanism in Aspergillus fumigatus
}

Grainne O'Keeffe 1 , Stephen Hammel', Rebecca A Owens ${ }^{1}$, Thomas M Keane², David A Fitzpatrick', Gary W Jones ${ }^{1}$ and Sean Doyle ${ }^{1^{*}}$

\begin{abstract}
Background: Aspergillus fumigatus produces a number of secondary metabolites, one of which, gliotoxin, has been shown to exhibit anti-fungal activity. Thus, A. fumigatus must be able to protect itself against gliotoxin. Indeed one of the genes in the gliotoxin biosynthetic gene cluster in A. fumigatus, gliT, is required for self-protection against the toxin- however the global self-protection mechanism deployed is unclear. RNA-seq was employed to identify genes differentially regulated upon exposure to gliotoxin in A. fumigatus wild-type and A. fumigatus $\Delta$ gliT, a strain that is hypersensitive to gliotoxin.

Results: Deletion of $A$. fumigatus gliT resulted in altered expression of 208 genes ( $\log _{2}$ fold change of 1.5) when compared to A. fumigatus wild-type, of which 175 genes were up-regulated and 33 genes were down-regulated. Expression of 164 genes was differentially regulated ( $\log _{2}$ fold change of 1.5) in A. fumigatus wild-type when exposed to gliotoxin, consisting of 101 genes with up-regulated expression and 63 genes with down-regulated expression. Interestingly, a much larger number of genes, 1700, were found to be differentially regulated ( $\log _{2}$ fold change of 1.5) in A. fumigatus $\Delta$ gliT when challenged with gliotoxin. These consisted of 508 genes with up-regulated expression, and 1192 genes with down-regulated expression. Functional Catalogue (FunCat) classification of differentially regulated genes revealed an enrichment of genes involved in both primary metabolic functions and secondary metabolism. Specifically, genes involved in gliotoxin biosynthesis, helvolic acid biosynthesis, siderophore-iron transport genes and also nitrogen metabolism genes and ribosome biogenesis genes underwent altered expression. It was confirmed that gliotoxin biosynthesis is induced upon exposure to exogenous gliotoxin, production of unrelated secondary metabolites is attenuated in A. fumigatus $\Delta$ gliT, while quantitative proteomic analysis confirmed disrupted translation in A. fumigatus $\Delta$ gliT challenged with exogenous gliotoxin.

Conclusions: This study presents the first global investigation of the transcriptional response to exogenous gliotoxin in A. fumigatus wild-type and the hyper-sensitive strain, $\Delta$ gliT. Our data highlight the global and extensive affects of exogenous gliotoxin on a sensitive strain devoid of a self-protection mechanism and infer that GliT functionality is required for the optimal biosynthesis of selected secondary metabolites in A. fumigatus.
\end{abstract}

Keywords: Gliotoxin, RNA-seq, Transcriptome, Secondary metabolism, Fungal proteomics

\footnotetext{
* Correspondence: sean.doyle@nuim.ie

'Department of Biology, National University of Ireland Maynooth, Maynooth, Co. Kildare, Ireland

Full list of author information is available at the end of the article
} 


\section{Background}

Gliotoxin, a non-ribosomally synthesised peptide produced by Aspergillus fumigatus and related fungi, is redox-active, depletes cellular glutathione (GSH), causes DNA damage and protein modification, and is consequently capable of inhibiting mammalian, fungal and bacterial cell growth [1-5]. Conversely, it has also been demonstrated that gliotoxin presence protects against $\mathrm{H}_{2} \mathrm{O}_{2}$-induced oxidative stress in A. fumigatus and that gliotoxin can substitute for peroxiredoxin in mammalian cells to protect against similar oxidative stresses [6,7]. In A. fumigatus, gliotoxin biosynthesis is encoded by a 13-gene cluster, $g l i$, and it has been demonstrated that gliotoxin effects induction of many genes within the gli cluster in a positive feedback manner $[2,8,9]$. Thus, gliotoxin presence induces gli cluster activation via $g l i Z$, a $\mathrm{Zn}_{2} \mathrm{Cys}_{6}$ binuclear transcription factor, and deletion of $g l i Z$ abolishes gliotoxin biosynthesis [10]. Moreover, Forseth et al. [11] revealed that an additional nine metabolites, dependent on gliZ presence, are produced consequent to gliotoxin biosynthetic pathway functionality in A. fumigatus.

Gliotoxin exposure has been shown by qRT-PCR to either activate or induce increased expression of all genes in the gli cluster, as especially observed for A. fumigatus $\triangle g l i P$, deficient in the non-ribosomal peptide synthetase which mediates cyclo-L-Phe-L-Ser formation $[9,12]$. Others have shown induction of gliG (a glutathione $S$-transferase), gliA (an MFS transporter) and gliT upon exposure of A. fumigatus wild-type to gliotoxin, by Northern analysis [2]. However, definitive evidence of concomitant increased de novo gliotoxin production has not been forthcoming. Relatedly, it has been shown that transformation with $A$. fumigatus gliA confers resistance against exogenous gliotoxin upon Leptosphaeria maculans [13] while deletion of gliA in A. fumigatus renders it less resistant to exogenous gliotoxin [14], and Schrettl et al. [2] were the first to demonstrate increased GliT abundance by 2D-PAGE/MALDI-ToF analysis in A. fumigatus upon exposure to exogenous gliotoxin. However, within A. fumigatus, gliotoxin biosynthesis must be controlled to avoid manifestation of the deleterious affects of this reactive metabolite.

Since the original observations that self-protection against gliotoxin was largely mediated by the enzyme GliT, a gliotoxin oxidoreductase $[2,15]$, it has subsequently been found that other organisms contain similar enzymes which facilitate self-protection against related epipolythiodioxopiperazines $[16,17]$. Indeed, in the bacterium, Streptomyces clavuligerus, it has been demonstrated that an oxidoreductase, HlmI, confers self-protection against the disulfide-bridge-containing, non-ribosomal peptide, holomycin [17]. Interestingly, an RNA methyltransferase, Hom12 in Yersinia ruckeri, also enables self-protection against holomycin, since Hom12 deletion results in acquisition of a holomycin-sensitive phenotype [16]. This clearly infers that self-protection against redox-active non-ribosomal peptides is a multi-faceted process, yet few studies have attempted molecular dissection of the process. Carberry et al. [5] revealed significantly elevated GSH levels in A. fumigatus $\Delta g l i T$, and speculated about exacerbation of gliotoxin toxicity, resulting from formation of the dithiol form of gliotoxin, consequent to this apparent dysregulation in the level of an important cellular reductant. Indeed, the apparent resistance of Saccharomyces cerevisiae $\Delta g s h 1$, which exhibits significantly attenuated GSH levels, to exogenous gliotoxin supported this hypothesis. Interestingly, these authors also observed that S. cerevisiae $\Delta$ sod1 and $\Delta y a p 1$ were hypersensitive to exogenous gliotoxin, suggesting that a deficient oxidative stress response sensitizes this organism to gliotoxin. Coleman et al. [4] further revealed that both Candida albicans and Cryptococcus neoformans were sensitive to gliotoxin exposure, however apart from an elegant demonstration of membrane damage consequent to gliotoxin exposure, no mechanistic basis of the anti-fungal effect of gliotoxin was forthcoming.

It is somewhat surprising that the concept of selfprotection against gliotoxin, in fungi capable of its biosynthesis, has received scant attention since the discovery of gliotoxin in 1936 - given the reactive nature of this disulfide-containing metabolite. However the availability of powerful new technologies such as RNA-seq [18-21], provides us with a tool to address this information deficient. Consequently, we present here the first exploration of the global transcriptomic response of both $A$. fumigatus wild-type and $\Delta g l i T$ to exogenous gliotoxin, which illuminates the important role played by gliT in mediating control of the cellular systems in the presence of this reactive metabolite.

\section{Results}

Deletion of gliT results in altered expression of over 200 genes involved in many functions in $A$. fumigatus

As deletion of $A$. fumigatus gliT renders the strain sensitive to exogenous gliotoxin $[2,15]$, to achieve a better understanding of the self-protection against gliotoxin provided by $A$. fumigatus gliT, high throughput RNA sequencing analysis was carried out. An average of 9312 transcripts were expressed in A. fumigatus wild-type and $\Delta g l i T$ (available from the European Nucleotide Archive under accession ERP001382), which is in accordance with other RNA-seq investigations of the A. fumigatus transcriptome [18,22]. A comparison of A. fumigatus wild-type and $\Delta g l i T$ revealed that the deletion of gliT resulted in the significant $(p<0.05)$ dysregulated expression of 208 genes, consisting of 175 up-regulated genes while 33 genes were significantly down-regulated $(p<0.05)$ (Figure $1 \mathrm{~A}$, Additional file 1 ). The differentially regulated genes were classified according to the Functional Catalogue (FunCat) 


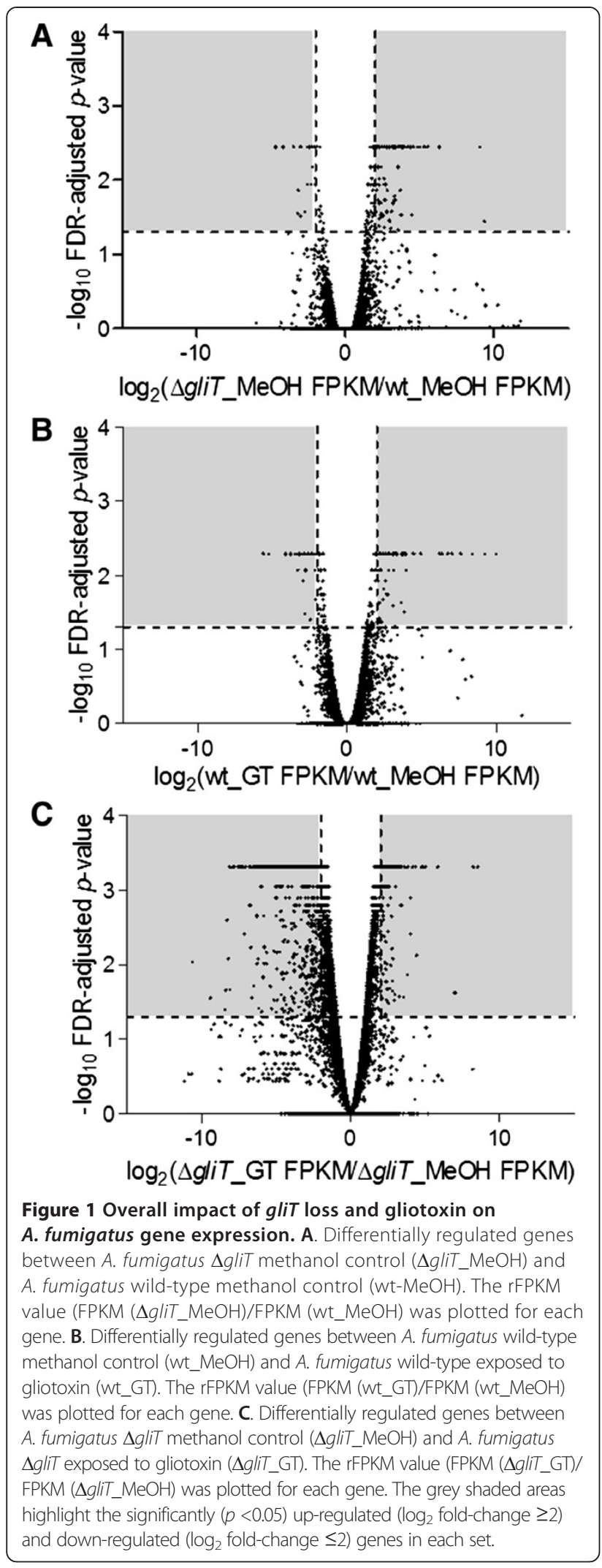

[23] and KEGG pathways [24] to identify the functions of these genes. Following deletion of gliT, $132^{\text {nd }}$ level, $193^{\text {rd }}$ level and $124^{\text {th }}$ level FunCat categories were overrepresented in the up-regulated gene set, compared to $32^{\text {nd }}$ level, $103^{\text {rd }}$ level and $34^{\text {th }}$ level FunCat categories enriched in the down-regulated gene set (Additional file 2: Tables S1 and S2). Categories over-represented in the up-regulated gene set include; secondary metabolism, transport and detoxification, while genes involved in C-compound and carbohydrate metabolism were enriched in the downregulated gene set (Figure 2). Additionally, 11 KEGG pathways were over-represented in the up-regulated gene set, while genes involved in 13 KEGG pathways were enriched in the down-regulated gene set (Additional file 2: Tables S3 and S4). Carbohydrate and lipid metabolism were overrepresented in the up-regulated gene set, while amino acid metabolism was enriched in the down-regulated gene set. Genes involved in secondary metabolite biosynthesis were enriched in both the up- and down-regulated gene sets.

\section{Gliotoxin exposure alters the regulation of hundreds of} genes in both $A$. fumigatus wild-type and $\Delta$ gliT

Following $A$. fumigatus wild-type exposure to exogenous gliotoxin, 164 genes were significantly differentially regulated $(p<0.05)$ compared to control conditions (Figure 1B). Of these 164 genes, 101 genes were up-regulated when A. fumigatus wild-type was exposed to gliotoxin, while 63 genes were down-regulated (Additional file 1). In contrast, when A. fumigatus $\Delta g l i T$ was exposed to exogenous gliotoxin, 1,700 genes were significantly differentially regulated $(p<0.05)$ (Figure $1 C)$, whereby expression of 508 genes was up-regulated in response to gliotoxin exposure and 1192 genes down-regulated (Additional file 1). Given the large transcriptomic remodelling observed in A. fumigatus $\Delta g l i T$ following exogenous gliotoxin exposure, cell viability was assessed at $85 \%$. Although a significant decrease $(p=0.0019)$ in the cell viability of A. fumigatus $\Delta$ gliT exposed to exogenous gliotoxin, compared with the methanol control (97\%) (Additional file 2: Figure S1), gross cell death was not observed.

\section{Functional characterisation of differentially regulated} genes in A. fumigatus wild-type and $\Delta$ gliT following exogenous gliotoxin exposure

In classifying the differentially regulated genes in A. fumigatus wild-type upon exposure to exogenous gliotoxin, $92^{\text {nd }}$ level, $173^{\text {rd }}$ level and $114^{\text {th }}$ level FunCat categories, respectively, were over-represented in the up-regulated gene set compared to $122^{\text {nd }}$ level, $173^{\text {rd }}$ level and $84^{\text {th }}$ level categories in the down-regulated gene set (Additional file 2: Tables S5 and S6). Comparatively, in A. fumigatus $\Delta$ gliT exposed to exogenous gliotoxin, a greater number of FunCat categories were over-represented. Here, 24. $2^{\text {nd }}$ level, $233^{\text {rd }}$ level and $124^{\text {th }}$ level FunCat 


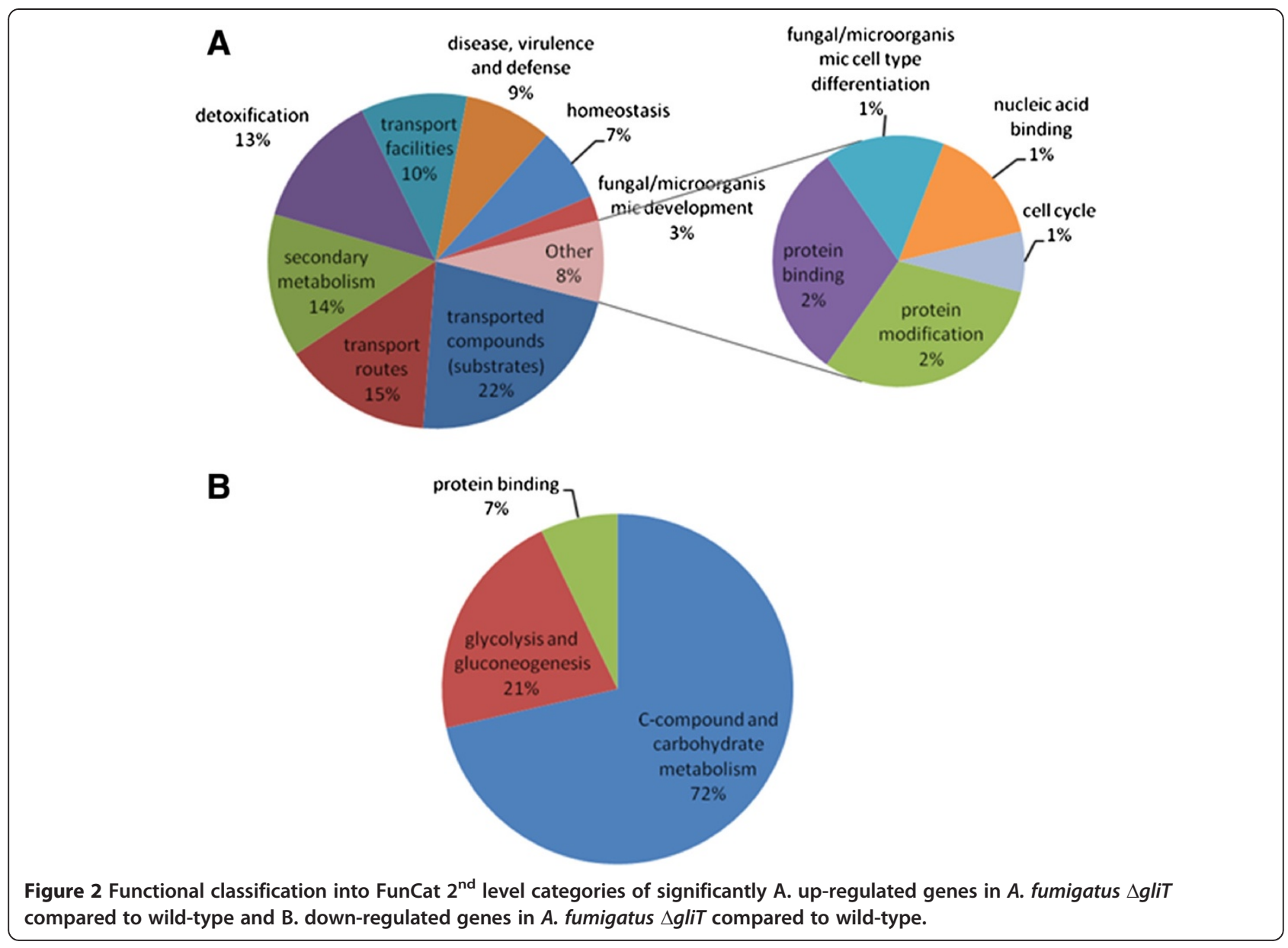

categories, respectively, were over-represented in the up-regulated gene set compared to $422^{\text {nd }}$ level, $843^{\text {rd }}$ level and $394^{\text {th }}$ level categories in the down-regulated gene set (Additional file 2: Tables S7 and S8). A. fumigatus wild-type exposure to exogenous gliotoxin results in an over-representation of up-regulated expression of genes involved in secondary metabolism, transport (particularly siderophore-iron transport), detoxification processes and homeostasis processes as well as others (Figure 3). Of the down-regulated genes, some of the categories which were enriched included amino acid metabolism, carbohydrate metabolism and complex cofactor/cosubstrate/vitamin binding (Figure 3). Loss of gliT in combination with exogenous gliotoxin exposure results in the dysregulation of a large number of processes within the cell. It resulted in an over-representation of upregulated genes involved in stress response, ribosome biogenesis and translation, and of down-regulated genes involved in metabolism of cysteine, nitrogen, sulphur and selenium metabolism, RNA synthesis, transport (including siderophore-iron transport), homeostasis and cellular import (Figure 4).
In combination with the identified FunCat categories, 2 KEGG pathways were over-represented in the up-regulated gene set, and 13 KEGG pathways in the downregulated gene set of $A$. fumigatus wild-type upon exogenous gliotoxin exposure were over-represented (Additional file 2: Tables S9 and S10). Pathways involved in xenobiotic biodegradation and metabolism were enriched in the up-regulated gene set, while in the down-regulated gene set, pathways involved in the biosynthesis of secondary metabolites, glycolysis/gluconeogenesis and amino acid metabolism are over-represented. In A. fumigatus $\Delta$ gliT exposed to exogenous gliotoxin, 18 KEGG pathways were over-represented in the up-regulated gene set compared to 38 KEGG pathways in the downregulated gene set (Additional file 2: Tables S11 and S12). Among the KEGG pathways over-represented in the up-regulated gene set were pathways involved in carbohydrate metabolism, translation and selenoamino acid metabolism. In the down-regulated gene set, some of the pathways over-represented included; biosynthesis of secondary metabolites, starch and sucrose metabolism, and amino acid metabolism. 

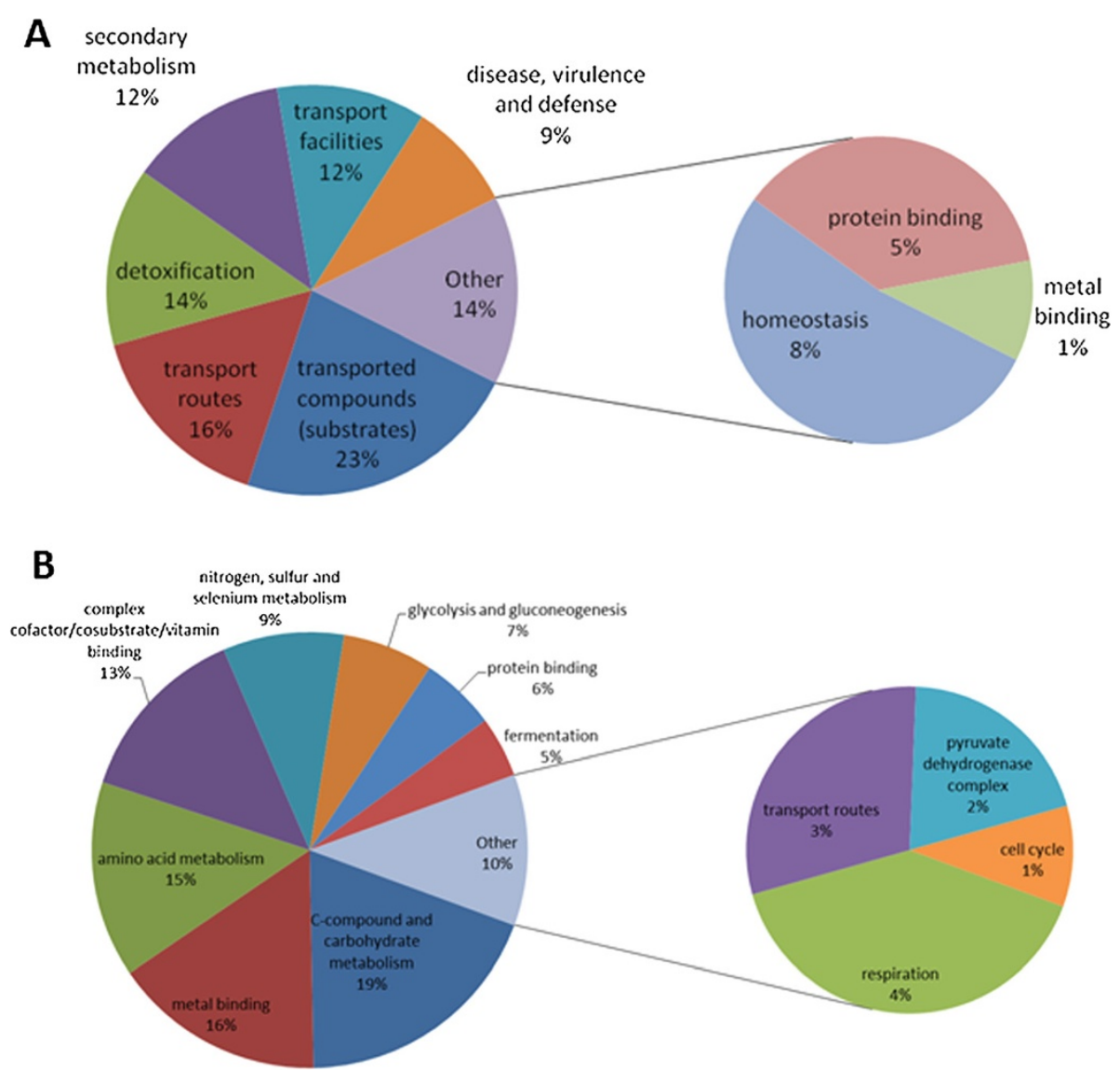

Figure 3 Functional classification into FunCat $2^{\text {nd }}$ level categories of significantly A. up-regulated genes in A. fumigatus wild-type exposed to gliotoxin compared to the $\mathrm{MeOH}$ control and $\mathrm{B}$. down-regulated genes in A. fumigatus wild-type exposed to gliotoxin compared to the $\mathrm{MeOH}$ control.

Exogenous gliotoxin causes dysregulation of gliotoxin biosynthesis cluster gene expression in both $A$. fumigatus wild-type and $\Delta$ gliT

Close inspection of the 13-gene gliotoxin biosynthesis cluster [2,3], revealed that exogenous gliotoxin caused the dysregulated expression of a number of gliotoxin biosynthetic genes in both A. fumigatus wild-type and $\Delta g l i T$ (Table 1). Upon exposure to exogenous gliotoxin, five out of the 13 genes in the cluster were significantly up-regulated in A. fumigatus wild-type. Expression of A. fumigatus gliZ was up-regulated $\log _{2}$ 3.2-fold, gliP $\log _{2} 6.2$ fold, while gliA and gliF were up-regulated $\log _{2} 10$ - and $\log _{2}$ 6.7-fold, respectively. Expression of gliT, which confers protection against exogenous gliotoxin $[2,15]$, was up-regulated $\log _{2} 9.2$-fold. Increased expression of the remaining genes in the gliotoxin biosynthetic cluster was also observed in A. fumigatus wild-type upon exogenous gliotoxin exposure, however altered expression was not significant.

Upon A. fumigatus $\Delta g l i T$ exposure to exogenous gliotoxin, dysregulated expression of gliotoxin biosynthetic genes was also observed (Table 1). As in A. fumigatus wild-type, gliP, gliA and gliF expression was also upregulated in $\Delta g l i T$ upon exogenous gliotoxin addition. A. fumigatus gliP was up-regulated $\log _{2} 3.4$-fold, gliA was up-regulated $\log _{2} 8.5$-fold and A. fumigatus gliF was up-regulated $\log _{2} 4.7$-fold. Additionally, A. fumigatus gliM was up-regulated $\log _{2} 6.9$-fold in A. fumigatus $\Delta g l i T$ exposed to exogenous gliotoxin, which was not observed in A. fumigatus wild-type upon gliotoxin exposure. Of the remaining gliotoxin biosynthetic genes, while not significant, with the exception of A. fumigatus gliZ and gliI which did not exhibit altered expression, and gliJ which appeared to undergo down-regulated expression, increased expression of the remaining genes was observed in A. fumigatus $\Delta g l i T$ upon exposure to exogenous gliotoxin. Quantitative real-time PCR (qRT-PCR) analysis of the expression of A. fumigatus gliZ confirmed the up-regulation in A. fumigatus wild-type exposed to exogenous gliotoxin compared to the control, however it also showed increased $g l i Z$ expression in $\Delta g l i T$ in response to gliotoxin (Figure 5), 

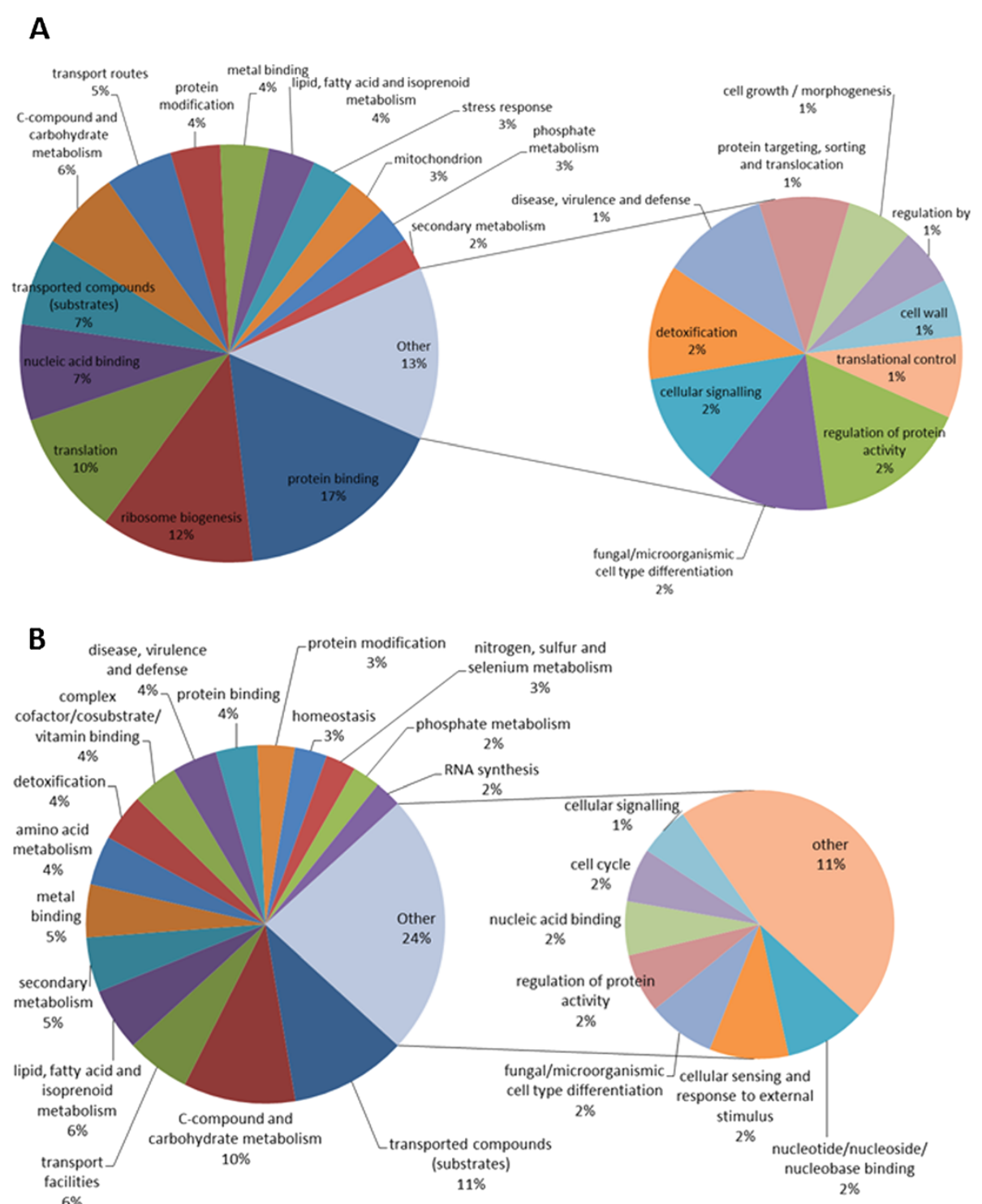

Figure 4 Functional classification into FunCat $2^{\text {nd }}$ level categories of significantly A. up-regulated genes in A. fumigatus $\Delta$ gliT exposed to gliotoxin compared to the $\mathrm{MeOH}$ control and B. down-regulated genes in A. fumigatus $\Delta$ gliT exposed to gliotoxin compared to the $\mathrm{MeOH}$ control.

which was not observed in the RNA-seq analysis, possibly due to the sensitivities of the different techniques. qRT-PCR analysis of the expression of A. fumigatus gliA in both A. fumigatus wild-type and A. fumigatus $\triangle g l i T$ in both the absence and presence of exogenous gliotoxin confirmed the up-regulation in response to exogenous gliotoxin (Figure 5).

The impact of gliotoxin on the expression of the gliotoxin biosynthetic genes is in accordance with that observed in other studies using different techniques [2,9]. Moreover, feeding experiments with $\left[{ }^{13} \mathrm{C}\right]$-phenylalanine herein confirm, for the first time, that gliotoxin biosynthesis is actually induced by exogenous gliotoxin. A significant increase $(p=0.0295)$ was observed in the amount of $\left[{ }^{13} \mathrm{C}\right]$-gliotoxin detectable in the wild-type A. fumigatus Af293 culture supernatants following exogenous gliotoxin addition compared to the methanol control (Figure 6).

\section{Exogenous gliotoxin results in down-regulation of secondary} metabolite gene cluster expression in $A$. fumigatus $\Delta$ gliT

FunCat classification of the altered gene expression in A. fumigatus wild-type and $\Delta g l i T$, respectively, revealed an enrichment of genes involved in secondary metabolism upon exogenous gliotoxin exposure. In A. fumigatus wild-type, expression of 16 genes which was up-regulated, and that of 3 genes down-regulated in response to exogenous gliotoxin, were classified by $2^{\text {nd }}$ level of FunCat as being involved in secondary metabolism (Additional file 2: 
Table $1 \mathrm{Log}_{2}$ (fold change) in the expression of the gliotoxin biosynthetic genes in $A$. fumigatus wild-type and $\Delta$ gliT exposed to exogenous gliotoxin

\begin{tabular}{|c|c|c|c|c|c|}
\hline \multirow[b]{2}{*}{ Gene } & \multirow[b]{2}{*}{ Gene name } & \multicolumn{2}{|c|}{ Wild-type_Glio v Wild-type_MeOH } & \multicolumn{2}{|c|}{$\Delta$ gliT_Glio v $\Delta$ gliT_MeOH } \\
\hline & & $\log _{2}$ (fold change) & q value & $\log _{2}$ (fold change) & q value \\
\hline AFUA_6G09630 & gliz & 3.165 & 0.005 & 0.465 & 0.525 \\
\hline AFUA_6G09640 & glil & 1.758 & 1.000 & -0.031 & 1.000 \\
\hline AFUA_6G09650 & glij & 2.250 & 0.383 & -2.816 & 1.000 \\
\hline AFUA_6G09660 & glip & 6.208 & 0.005 & 3.408 & 0.0005 \\
\hline AFUA_6G09670 & glic & 5.023 & 0.065 & 4.223 & 0.093 \\
\hline AFUA_6G09680 & glim & 11.737 & 0.769 & 6.986 & 0.024 \\
\hline AFUA_6G09690 & gliG & 7.956 & 0.253 & 8.199 & 0.254 \\
\hline AFUA_6G09700 & glik & 7.417 & 0.445 & 2.844 & 0.073 \\
\hline AFUA_6G09710 & gliA & 10.014 & 0.005 & 8.517 & 0.0005 \\
\hline AFUA_6G09720 & glin & 4.807 & 0.053 & 2.106 & 0.086 \\
\hline AFUA_6G09730 & glif & 6.758 & 0.005 & 4.745 & 0.0005 \\
\hline AFUA_6G09740 & glit & 9.248 & 0.005 & - & - \\
\hline AFUA_6G09745 & gliH & 5.075 & 0.128 & 2.688 & 0.237 \\
\hline
\end{tabular}

Tables S5 and S6). A larger number of genes were classified in this category in $A$. fumigatus $\Delta g l i T$ in response to exogenous gliotoxin presence, where 22 up-regulated genes and 103 down-regulated genes were observed (Additional file 2: Tables S7 and S8). A closer inspection of some of the secondary metabolite genes clusters revealed significant alterations in the expression of genes in the helvolic biosynthesis cluster [25] and the "supercluster" on chromosome 8 which encodes the biosynthetic pathways of a number of secondary metabolites, including fumitremorgin B, fumagillin and pseurotin A [26-29] (Table 2).

Helvolic acid, a triterpene, is encoded by a 9-gene cluster on chromosome 4 [25]. In A. fumigatus wild-type, exogenous gliotoxin results in the significant up-regulation of 2 genes, A. fumigatus osc3 ( $\log _{2} 3.28$-fold) and $s d r I$ ( $\log _{2}$ 3.22-fold) (Table 2). Conversely, in A. fumigatus $\Delta g l i T$ exposed to exogenous gliotoxin, osc3 was significantly down-regulated ( $\log _{2} 6.3$-fold) along with a predicted $O$-methyltransferase, AFUA_4G14580, which is significantly down-regulated ( $\log _{2} 2.7$-fold) (Table 2 ). In addition to this, expression of cyp5081B1 is completely abrogated in $A$. fumigatus $\Delta g l i T$ exposed to exogenous gliotoxin (Table 2). qRT-PCR analysis of $A$. fumigatus osc3 confirmed the observed down-regulation in $A$. fumigatus $\Delta g l i T$ and up-regulation of $A$. fumigatus osc3 in A. fumigatus wild-type exposed to exogenous gliotoxin (Figure 5).

Of the 69 genes in the "supercluster" on chromosome 8, expression of two genes is significantly down-regulated in $A$. fumigatus wild-type in response to exogenous gliotoxin (Table 2). However, in A. fumigatus $\Delta g l i T$, when exposed to exogenous gliotoxin, expression of 26 genes from the "supercluster" was down-regulated (Table 2). Closer inspection of the fumitremorgin $\mathrm{B}$ biosynthetic genes revealed that expression of the non-ribosomal peptide synthetase (NRPS), A. fumigatus ftmA [29], was significantly down-regulated ( $\log _{2} 2.78$-fold) in A. fumigatus wild-type in exogenous gliotoxin presence (Table 2). While in $A$. fumigatus $\triangle$ gliT, ftmA ( $\log _{2} 6.65$-fold), ftmC ( $\log _{2} 5.68$-fold), ftmD ( $\log _{2}$ 5.87-fold), ftmG ( $\log _{2} 6.52$-fold) and ftmI ( $\log _{2}$ 1.45-fold) expression was significantly down-regulated, and ftmE expression was completely inhibited in response to exogenous gliotoxin (Table 2). qRT-PCR analysis of $A$. fumigatus ftmA confirmed decreased expression in both wild-type and $\Delta g l i T$ following exposure to exogenous gliotoxin (Figure 5). Determination of the levels of the fumitremorgins and associated compounds in A. fumigatus wild-type and $\Delta g l i T$ cultured in secondary metaboliteinducing conditions (96 h in Czpaeks-Dox Broth) revealed significant alterations in the levels of a number of cognate metabolites. Specifically, brevianamide F levels were significantly increased in A. fumigatus $\Delta g l i T$ compared to wildtype $(p=0.0243)$, while the levels of both tryprostatin A and tryprostatin B were significantly decreased ( $p=0.008$ and $p=0.0453$ ) in $A$. fumigatus $\Delta g l i T$ compared to wildtype (Figure 7). There was no significant difference determined in the level of fumitremorgin $\mathrm{C}$ between A. fumigatus wild-type and $\Delta g l i T$ (Figure 7).

There was no significant dysregulation of the fumagillin biosynthetic genes in $A$. fumigatus wild-type in exogenous gliotoxin presence. However, when A. fumigatus $\Delta g l i T$ was exposed to exogenous gliotoxin, expression of 12 fumagillin biosynthetic genes in the cluster was significantly downregulated $(p<0.05)$ (Table 2$)$. Some of the down-regulated genes include a polyketide synthase (PKS) (A. fumigatus fma-PKS) [30] and a putative PKS (AFUA_8G00490) [27] which were down-regulated $\log _{2} 7.20$ - and $\log _{2} 5.65$-fold, 


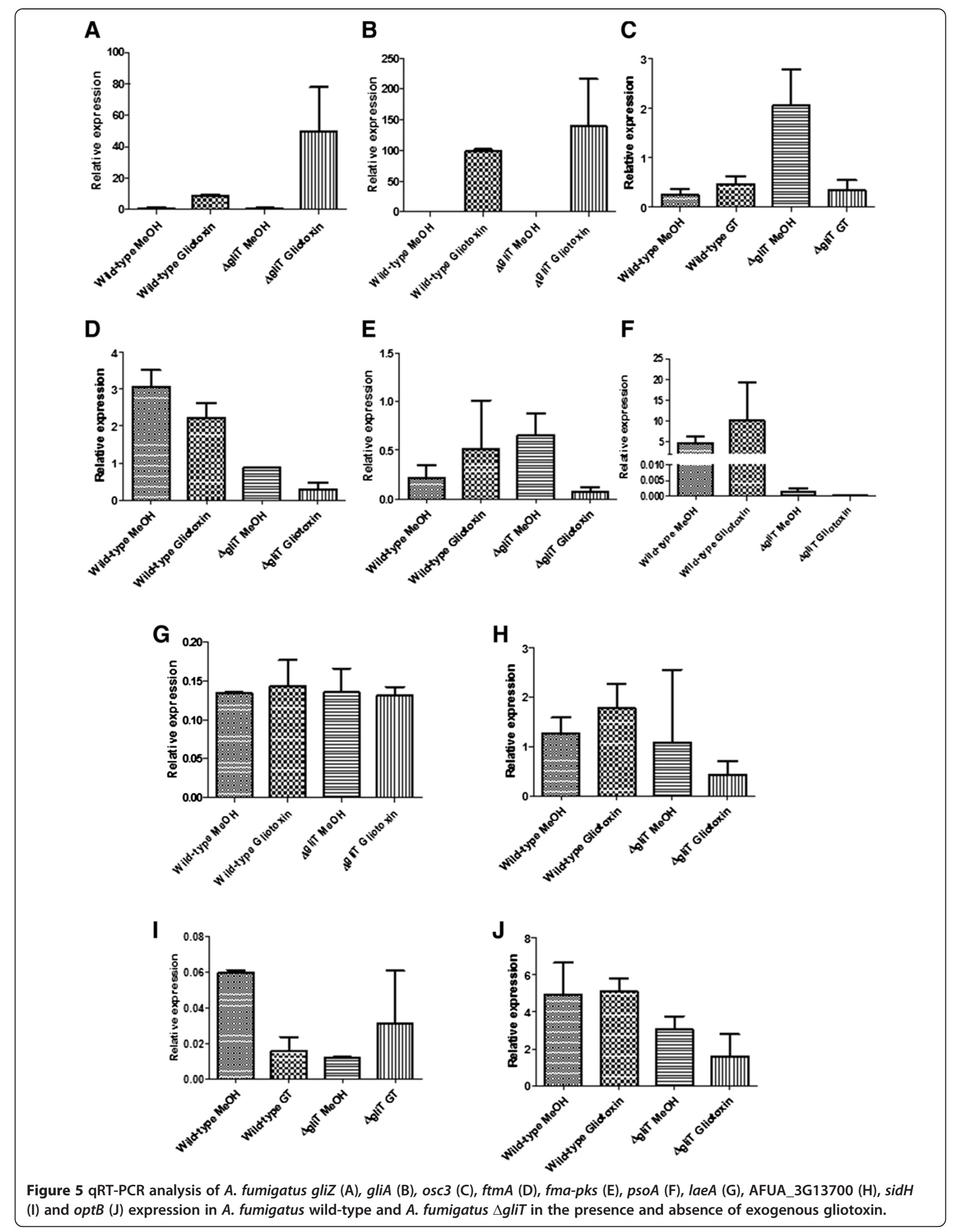




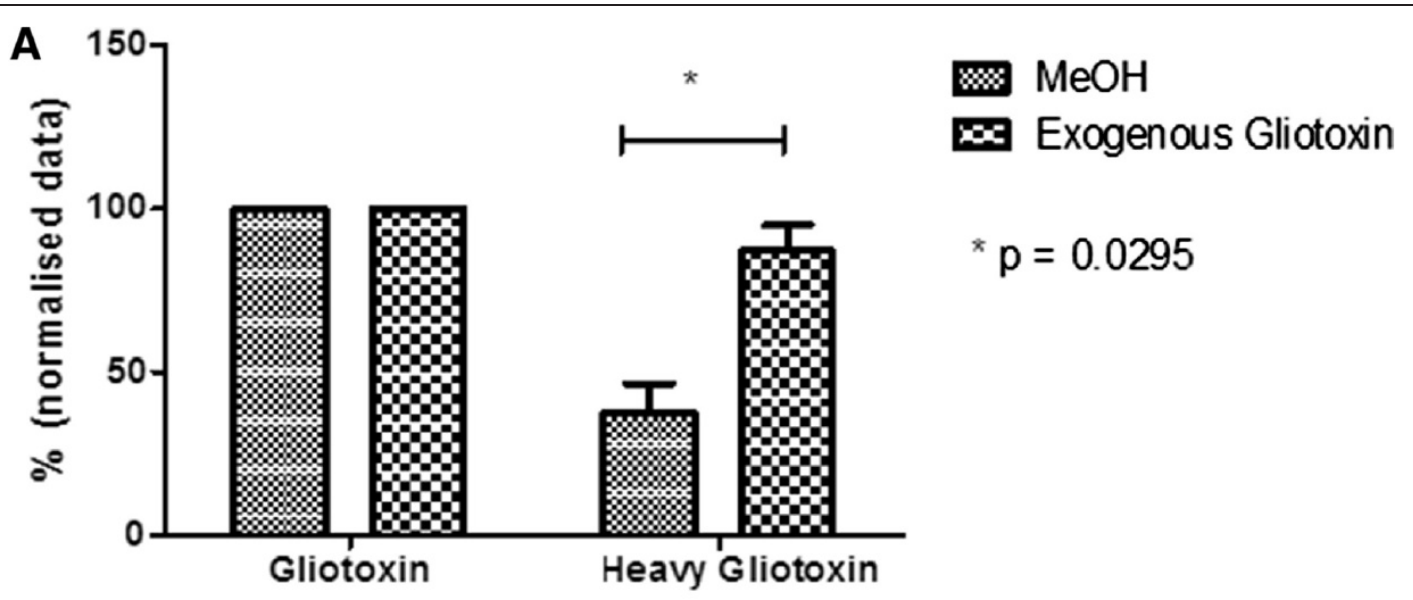

B

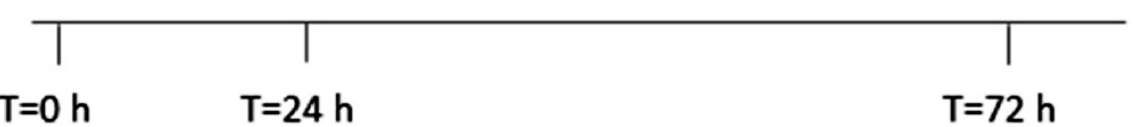

C

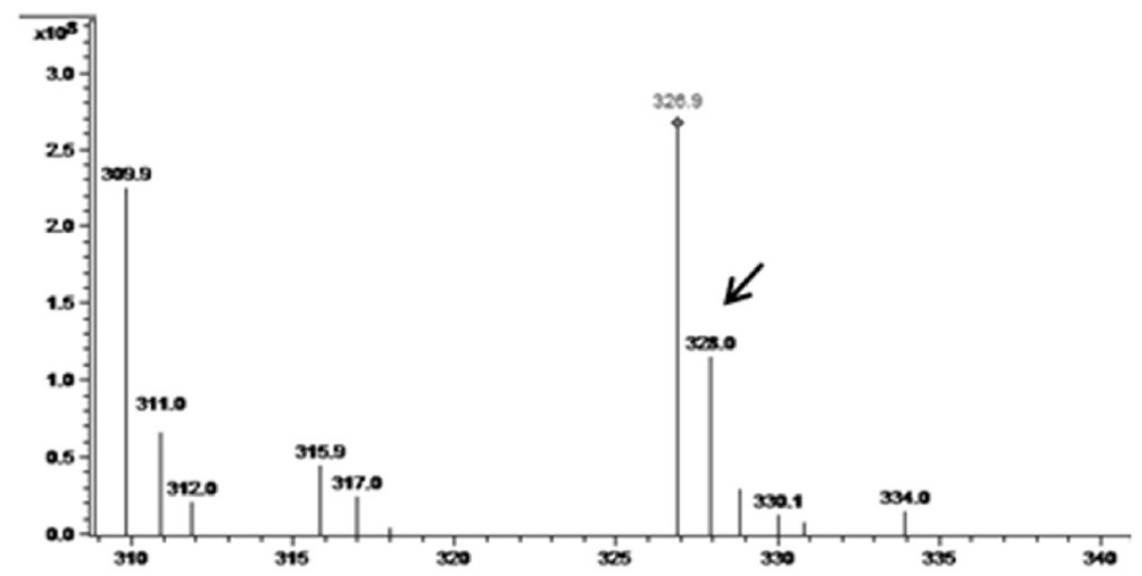

D

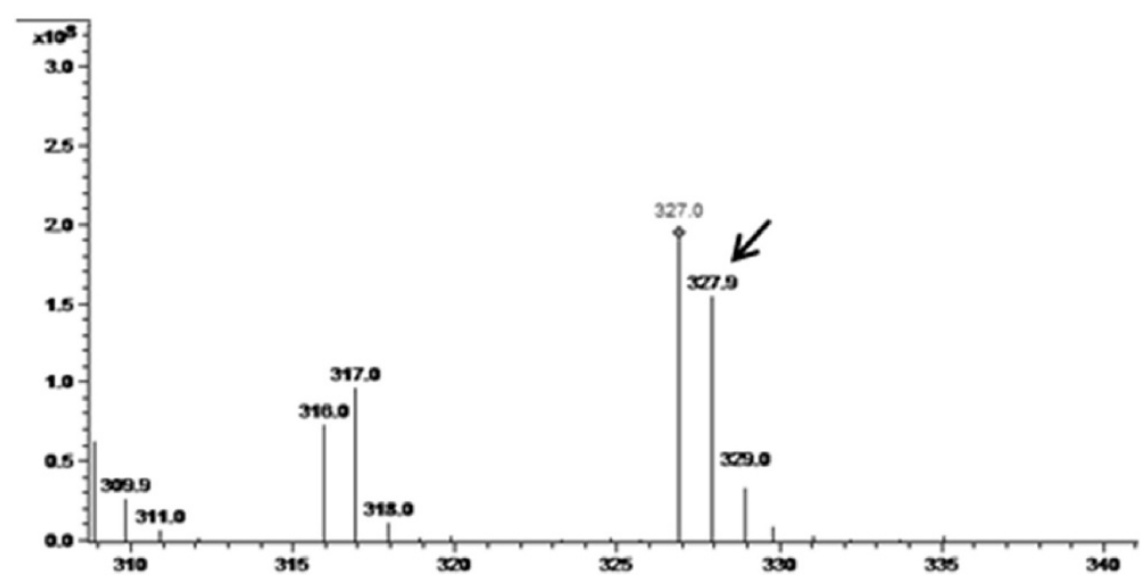

Figure 6 (See legend on next page.) 
(See figure on previous page.)

Figure 6 Gliotoxin biosynthesis is induced with exogenous gliotoxin addition. A. A significant increase $(p=0.0295)$ in the amount of de novo gliotoxin $\left(\left[{ }^{13} \mathrm{C}\right]\right.$-gliotoxin) produced following addition of exogenous gliotoxin was observed compared to the methanol control. B. Time-line of the experimental conditions. Cultures (duplicate) were inoculated at $T=0 \mathrm{~h}$. At $\mathrm{T}=24 \mathrm{~h},\left[{ }^{13} \mathrm{C}\right]$-phenylalanine was added along with gliotoxin $(5 \mathrm{\mu g} / \mathrm{ml}$ final) or methanol (solvent control). Culture supernatants were collected at $\mathrm{T}=72 \mathrm{~h}$ and organic extraction carried out prior to LC-MS/MS analysis. C. Spectrum showing gliotoxin $(\mathrm{m} / \mathrm{z} 327)$ and $\left[{ }^{13} \mathrm{C}\right]$-gliotoxin $(\mathrm{m} / \mathrm{z} 328)$ in cultures spiked with the methanol. D. Spectrum showing gliotoxin $(\mathrm{m} / \mathrm{z} 327)$ and $\left[{ }^{13} \mathrm{C}\right]$-gliotoxin $(\mathrm{m} / \mathrm{z} 328)$ in cultures spiked with exogenous gliotoxin. The black arrows highlight $\left[{ }^{13} \mathrm{C}\right]$-gliotoxin, where higher levels are observed in cultures induced by exogenous gliotoxin compared to the methanol control.

respectively, A. fumigatus metAP/fpaII expression was down-regulated $\log _{2}$ 5.20-fold and fma-TC/fmaA was down-regulated $\log _{2} 8.37$-fold. The decreased expression of $A$. fumigatus fma-PKS in A. fumigatus $\triangle$ gliT exposed to exogenous gliotoxin was confirmed by qRT-PCR analysis (Figure 5). As was the case for fumitremorgin B and fumagillin, none of the pseurotin A biosynthetic genes were differentially regulated in $A$. fumigatus wild-type in exogenous gliotoxin presence (Table 2). However, in A. fumigatus $\Delta$ gliT exposed to exogenous gliotoxin, with the exception of AFUA_8G00570, expression of all of the pseurotin A biosynthetic genes was significantly downregulated (Table 2). Expression of AFUA_8G00530 and $A$. fumigatus psoA/nrps14, the PKS-NRPS hybrid [28], were down-regulated $\log _{2} 7.45$ - and $\log _{2}$ 5.79-fold respectively, while $A$. fumigatus $p s o C$, psoD and elfB [31] expression was down-regulated $\log _{2} 7.41-, \log _{2} 6.78$ - and $\log _{2}$ 8.21fold respectively. qRT-PCR confirmed the decreased expression of $A$. fumigatus psoA/nrps14 in A. fumigatus $\triangle g l i T$ upon exogenous gliotoxin exposure (Figure 5). Relevantly, under secondary metabolite inducing growth conditions, production of fumagillin and pseurotin A was significantly reduced ( $p=0.0471$ and $p=0.0297$, respectively) in $A$. fumigatus $\Delta$ gliT compared to wild-type (Figure 7).

The methyltransferase, laeA, is a global regulator of secondary metabolism in A. fumigatus, which fully or partially regulates expression of multiple secondary metabolite gene clusters, including those encoding gliotoxin, helvolic acid, pseurotin A, fumagillin and fumitremorgin biosynthesis $[26,27,32,33]$. Although dysregulation of secondary metabolite gene expression in A. fumigatus $\Delta g l i T$ compared to $A$. fumigatus was observed after exposure to gliotoxin, lae $A$ expression was not differentially regulated in $A$. fumigatus wild-type in exogenous gliotoxin presence, but was significantly down-regulated $\left(p=0.015\right.$; $\log _{2} 1.31$ fold) in A. fumigatus $\Delta$ gliT under identical conditions. Although significantly down-regulated, it was outside the cut-off of $\log _{2} 1.5$-fold change. qRT-PCR analysis for A. fumigatus laeA did not show any differential regulation in either $A$. fumigatus wild-type or A. fumigatus $\Delta g l i T$ when challenged with exogenous gliotoxin (Figure 5 ). This suggests that laeA expression is not solely responsible for the altered expression of secondary metabolite genes, particularly in A. fumigatus $\Delta g l i T$ when it is exposed to exogenous gliotoxin. Expression of A. fumigatus gliT in $\triangle l a e A$ [32] exposed to exogenous gliotoxin for $3 \mathrm{~h}$ was analysed. A. fumigatus gliT expression increased following exogenous gliotoxin exposure in $\triangle l a e A$ (Figure 8) indicating that loss of laeA does not affect gliT expression in the presence of exogenous gliotoxin. Recently, the global regulatory velvet gene, $A$. fumigatus veA, has been implicated in secondary metabolite biosynthesis regulation, particularly with respect to gliotoxin [20] and fumagillin [22]. However, from the data presented here, A. fumigatus veA is not differentially regulated in either $A$. fumigatus wildtype or $A$. fumigatus $\triangle g l i T$ in exogenous gliotoxin presence. A. fumigatus gliT expression in A. fumigatus $\Delta v e A$ [34] was assessed following exposure to exogenous gliotoxin and was increased in $\triangle v e A$ in the presence of exogenous gliotoxin (Figure 8). A. fumigatus gliT expression was higher in the methanol control (Figure 8) compared to the other mutants under the same conditions. Expression of A. fumigatus gliT in $\Delta v e A$ grown in media only was lower than the methanol control (data not shown), suggesting that methanol induced increased gliT expression in A. fumigatus $\Delta v e A$. The increased gliT expression in A. fumigatus $\Delta v e A$ following methanol addition was only observed in this strain, and was not observed in other deletion strains generated in this background (e.g. $\Delta l a e A)$.

The expression of $A$. fumigatus gliT was also investigated in deletion mutants with abrogated fumitremorgin $\mathrm{B}$, fumagillin and pseurotin A production namely, $A$. fumigatus $\triangle f a p R, \triangle f m a A$ and $\triangle p s o A$, respectively [27]. As was the case in $A$. fumigatus laeA, expression of $A$. fumigatus gliT increased in these mutants following exogenous gliotoxin exposure (Figure 8) indicating that loss of production of these metabolites does not affect gliT expression.

\section{Siderophore-iron transport and siderophore biosynthesis is affected by exogenous gliotoxin and to a greater extent in $A$. fumigatus $\Delta$ gliT}

Functional classification of the differentially regulated gene set revealed an enrichment of genes involved in siderophore-iron transport in both A. fumigatus wildtype and $A$. fumigatus $\Delta$ gliT when exposed to exogenous gliotoxin. In $A$. fumigatus wild-type exposed to exogenous gliotoxin, expression of six siderophore-iron transport genes was up-regulated, while in A. fumigatus 
Table $2 \log _{2}$ (fold change) in helvolic acid, fumitremorgin, fumagillin and pseurotin A biosynthetic gene cluster expression in $A$. fumigatus wild-type and $\Delta$ gliT exposed to exogenous gliotoxin

\begin{tabular}{|c|c|c|c|c|c|}
\hline \multirow[b]{2}{*}{ Gene } & \multirow[b]{2}{*}{ Gene name } & \multicolumn{2}{|c|}{ Wild-type_Glio v Wild-type_MeOH } & \multicolumn{2}{|c|}{$\Delta g l i T \_G l i o$ v $\Delta g l i T \_M e O H$} \\
\hline & & $\log _{2}$ (fold change) & q value & $\log _{2}$ (fold change) & $q$ value \\
\hline \multicolumn{6}{|l|}{ Helvolic acid } \\
\hline AFUA_4G14770 & osc 3 & 3.279 & 0.005 & -6.296 & 0.010 \\
\hline AFUA_4G14780 & сур5081A1 & 2.425 & 0.076 & -6.515 & 0.092 \\
\hline AFUA_4G14790 & сур5081B1 & 1.993 & 0.228 & Absent $^{\mathrm{a}}$ & 0.0005 \\
\hline AFUA_4G14800 & $s d r 1$ & 3.219 & 0.049 & -7.163 & 0.293 \\
\hline AFUA_4G14810 & cyp5081D1 & 2.633 & 0.200 & -3.952 & 0.053 \\
\hline AFUA_4G14820 & - & 2.701 & 0.057 & -5.964 & 0.063 \\
\hline AFUA_4G14830 & сур5081C1 & 0.470 & 1.000 & -4.229 & 1.000 \\
\hline AFUA_4G14840 & - & 1.542 & 1.000 & -2.941 & 1.000 \\
\hline AFUA_4G14850 & - & 0.960 & 0.761 & -2.702 & 0.021 \\
\hline \multicolumn{6}{|c|}{ Fumitremorgin B } \\
\hline AFUA_8G00170 & $\mathrm{ftmA}$ & -2.777 & 0.008 & -6.649 & 0.028 \\
\hline AFUA_8G00190 & $\mathrm{ftmC}$ & -1.278 & 0.205 & -5.681 & 0.0005 \\
\hline AFUA_8G00200 & $\mathrm{ftmD}$ & -1.263 & 0.181 & -5.870 & 0.0005 \\
\hline AFUA_8G00210 & ftmPT1 & -1.456 & 0.310 & -8.843 & 0.092 \\
\hline AFUA_8G00220 & $\mathrm{ftmE}$ & -0.614 & 1.000 & Absent $^{a}$ & 0.0005 \\
\hline AFUA_8G00230 & $\mathrm{ftmF}$ & -0.474 & 0.901 & -10.867 & 0.293 \\
\hline AFUA_8G00240 & $\mathrm{ftmG}$ & 0.611 & 0.844 & -6.519 & 0.019 \\
\hline AFUA_8G00250 & $\mathrm{ftmPT2}$ & 0.581 & 0.844 & -7.300 & 0.074 \\
\hline AFUA_8G00260 & $\mathrm{ftml}$ & 0.031 & 0.997 & -1.455 & 0.039 \\
\hline \multicolumn{6}{|l|}{ Fumagillin } \\
\hline AFUA_8G00370 & fma-PKS & 0.396 & 0.946 & -7.287 & 0.0005 \\
\hline AFUA_8G00380 & $f m a-A T$ & 0.264 & 0.968 & -9.364 & 0.074 \\
\hline AFUA_8G00390 & - & 0.220 & 0.976 & -7.902 & 0.0005 \\
\hline AFUA_8G00400 & - & -0.025 & 0.997 & -8.094 & 0.022 \\
\hline AFUA_8G00410 & metAP/fpall & -0.113 & 0.987 & -5.196 & 0.0005 \\
\hline AFUA_8G00420 & fapR/fumR & -0.284 & 0.957 & -5.826 & 0.0005 \\
\hline AFUA_8G00430 & - & 0.265 & 0.969 & -7.093 & 0.0005 \\
\hline AFUA_8G00440 & psoF & 0.005 & 0.998 & -5.426 & 0.0005 \\
\hline AFUA_8G00460 & fpal & -0.301 & 0.919 & -0.282 & 0.633 \\
\hline AFUA_8G00470 & fmaE & -0.176 & 0.968 & -0.271 & 0.636 \\
\hline AFUA_8G00480 & fmaF & 0.427 & 0.919 & -7.258 & 0.0005 \\
\hline AFUA_8G00490 & - & 0.306 & 0.959 & -5.649 & 0.007 \\
\hline AFUA_8G00500 & - & -0.047 & 0.997 & -6.780 & 0.002 \\
\hline AFUA_8G00510 & fmaG & 0.793 & 0.820 & -8.101 & 0.0005 \\
\hline AFUA_8G00520 & fma-TC/fmaA & 0.528 & 0.904 & -8.369 & 0.022 \\
\hline \multicolumn{6}{|l|}{ Pseurotin A } \\
\hline AFUA_8G00530 & $p s o B$ & 0.415 & 0.946 & -7.450 & 0.0005 \\
\hline AFUA_8G00540 & psoA/NRPS14 & 0.768 & 0.776 & -5.792 & 0.0005 \\
\hline AFUA_8G00550 & psoc & 0.292 & 0.964 & -7.413 & 0.0005 \\
\hline AFUA_8G00560 & psoD & 0.808 & 0.735 & -6.778 & 0.0005 \\
\hline
\end{tabular}


Table $2 \log _{2}$ (fold change) in helvolic acid, fumitremorgin, fumagillin and pseurotin A biosynthetic gene cluster expression in $A$. fumigatus wild-type and $\Delta$ gliT exposed to exogenous gliotoxin (Continued)

\begin{tabular}{lcccrr}
\hline AFUA_8G00570 & - & -0.271 & 0.965 & -8.059 & 0.064 \\
AFUA_8G00580 & psoE & 0.306 & 0.964 & -8.206 & 0.008 \\
AFUA_8G00590 & - & -0.982 & 0.239 & -1.430 & 0.009 \\
\hline
\end{tabular}

${ }^{\mathrm{a} G e n e}$ expression absent in $\Delta$ glit_Glio.

$\Delta g l i T$, expression of two siderophore-iron transport genes was up-regulated and 12 genes are down-regulated (Table 3). A. fumigatus sidF was de novo expressed in $A$. fumigatus wild-type upon exposure to exogenous gliotoxin. Expression of $A$. fumigatus mirD was up-regulated in both $A$. fumigatus wild-type ( $\log _{2} 2.57$-fold) and $A$. fumigatus $\Delta$ gliT ( $\log _{2}$ 3.07-fold) in exogenous gliotoxin presence. Interestingly, three genes that were significantly up-regulated in A. fumigatus wild-type exposed to exogenous gliotoxin were significantly down-regulated in A. fumigatus $\Delta g l i T$, when challenged with exogenous gliotoxin. A. fumigatus fre 7 was significantly up-regulated $\log _{2}$ 2.80 -fold in $A$. fumigatus wild-type, but significantly down-regulated $\log _{2}$ 2.77-fold in A. fumigatus $\Delta$ gliT. AFUA_3G13670 and AFUA_3G13700, significantly upregulated $\log _{2} 7.03$ - and $\log _{2} 7.62$-fold respectively, in $A$. fumigatus wild-type in exogenous gliotoxin presence, were significantly down-regulated upon exogenous gliotoxin
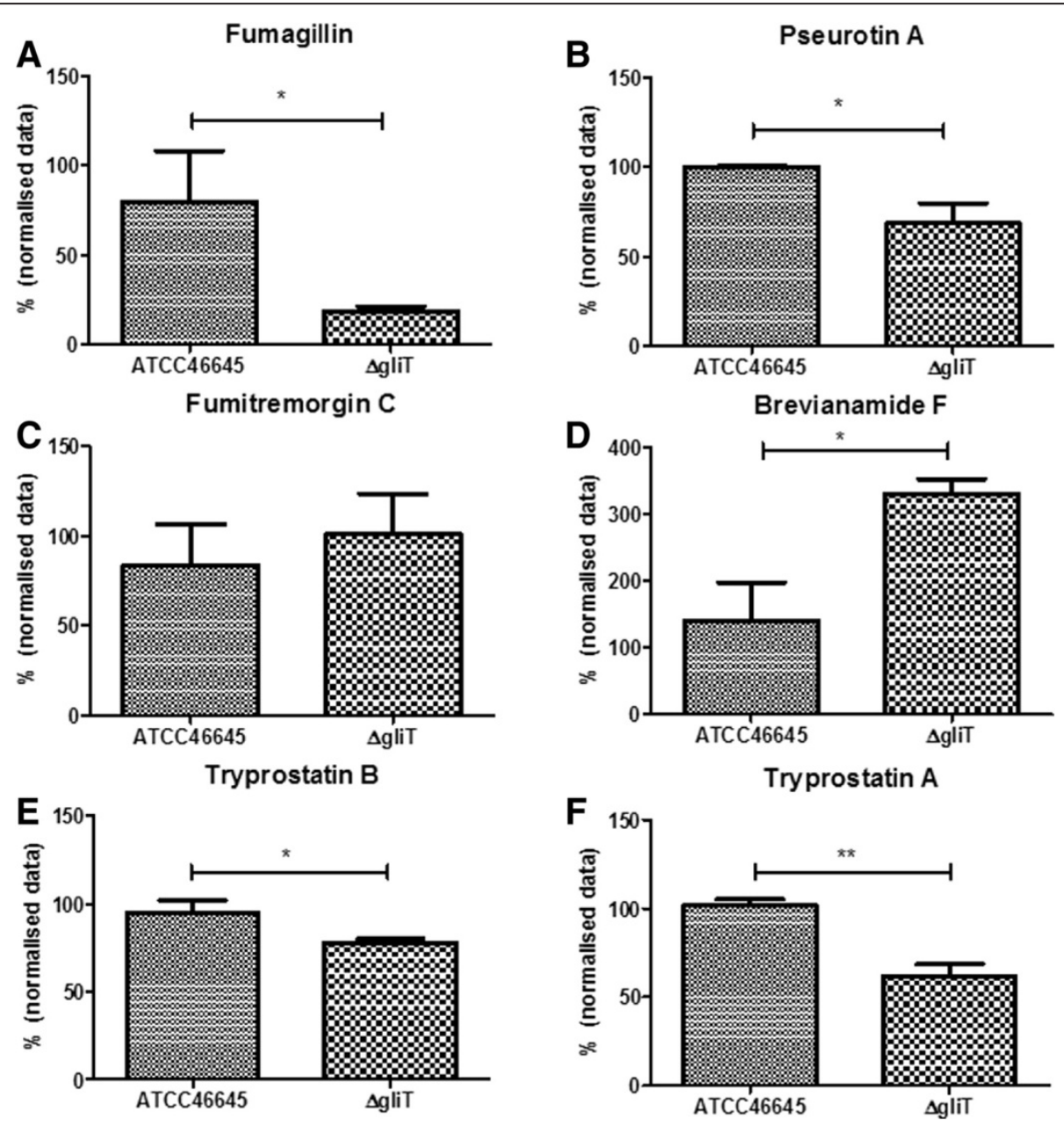

Figure 7 Levels of fumagillin, pseurotin A, fumitremorgin C, tryprostatin B and tryprostatin A in A. fumigatus wild-type and $\Delta$ gliT following $96 \mathrm{~h}$ growth under secondary metabolite inducing conditions. Fumagillin (A) and pseurotin A (B) production was significantly reduced in A. fumigatus $\Delta$ gliT compared to wild-type $\left({ }^{*} p=0.0471\right.$ and ${ }^{*} p=0.0297$, respectively). There was no significant difference in fumitremorgin $C$ (C) levels, while brevianamide F (D) levels were significantly increased ( $\left.*^{*} p=0.0243\right)$ in $\Delta$ gliT compared to wild-type. Tryprostatin B (E) and tryprostatin A (F) levels were significantly reduced in $\Delta$ gliT compared to wild-type (* $p=0.0453$ and ${ }^{* *} p=0.008$, respectively). 


\section{A}

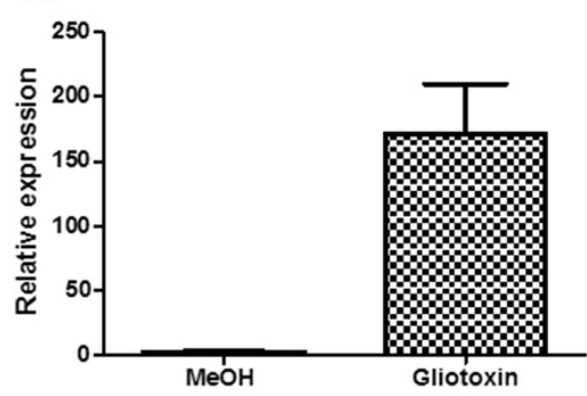

C
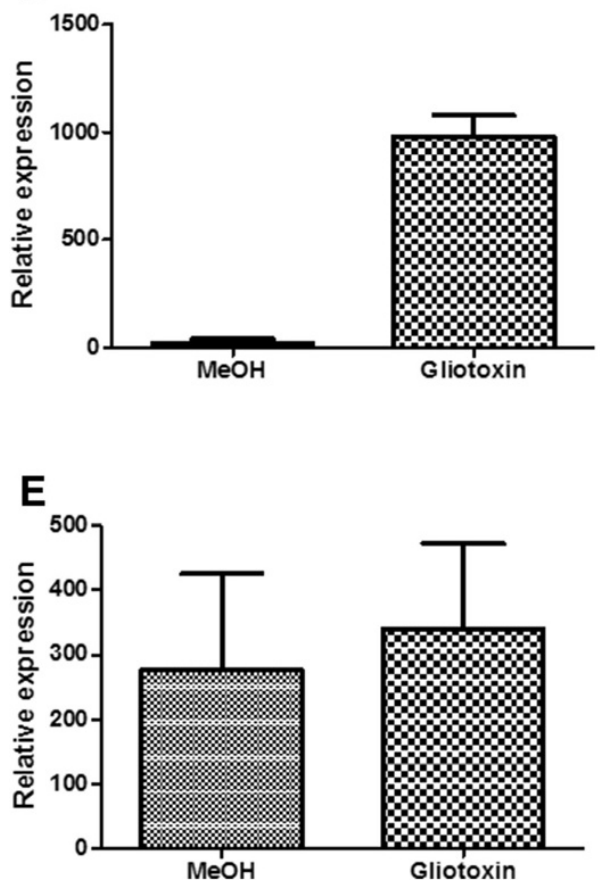

B

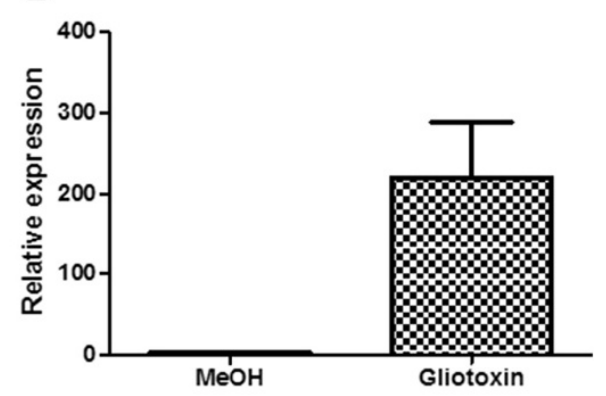

D

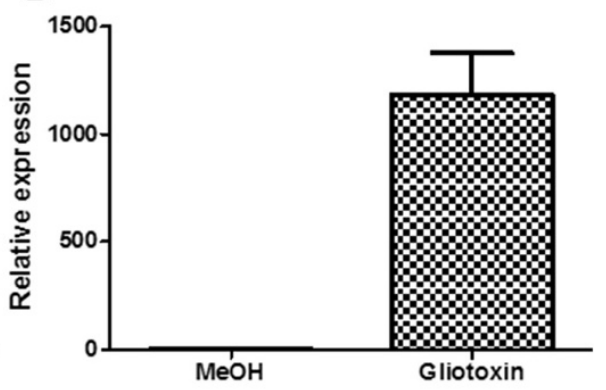

Figure 8 qRT-PCR analysis of $A$. fumigatus gliT expression in $A$. fumigatus $\triangle$ fmaA (A), $\Delta p s o A$ (B), $\Delta l a e A(C), \Delta$ fapR (D) and $\Delta v e A$ (E) exposed to exogenous gliotoxin.

exposure $\log _{2}$ 7.06- and $\log _{2} 7.82$-fold in A. fumigatus $\Delta g l i T$. Expression of AFUA_3G13700 in both A. fumigatus wild-type and $\Delta g l i T$ exposed to exogenous gliotoxin, was confirmed by qRT-PCR (Figure 5).

The observed differential regulation of genes involved in siderophore-iron transport prompted us to review whether expression of siderophore biosynthetic genes [35] was affected by exogenous gliotoxin in A. fumigatus wildtype and $A$. fumigatus $\Delta g l i T$. Indeed we have already noted that $s i d F$ expression, a transacylase that transfers anhydromevalonyl to hydroxyornithine during extracellular siderophore biosynthesis [36], was activated in A. fumigatus wild-type following exogenous gliotoxin exposure. In A. fumigatus $\Delta g l i T$, expression of two genes involved in siderophore biosynthesis was differentially regulated in response to exogenous gliotoxin (Table 3). A. fumigatus $s i d H$, involved in providing the anhydromevalonyl-CoA moiety for extracellular siderophore biosynthesis [37], was de novo expressed while A. fumigatus sidA, which is required for the first step of siderophore biosynthesis [38], was significantly up-regulated $\log _{2} 2.16$-fold $(p=0.0005)$. qRT-PCR analysis of the expression of $A$. fumigatus sidH confirmed the increased expression in A. fumigatus $\Delta g l i T$ following exogenous gliotoxin exposure (Figure 5).

\section{Nitrogen metabolism is down-regulated in A. fumigatus} $\Delta$ gliT in response to exogenous gliotoxin

In A. fumigatus $\Delta g l i T$ exposed to exogenous gliotoxin, expression of 55 genes involved in nitrogen metabolism was down-regulated, while expression of 4 genes was 
Table $3 \log _{2}$ (fold change) in siderophore-iron transport gene and siderophore biosynthetic gene expression in A. fumigatus wild-type and $\Delta$ gliT exposed to exogenous gliotoxin

\begin{tabular}{|c|c|c|c|c|c|}
\hline \multirow[b]{2}{*}{ Gene } & \multirow[b]{2}{*}{ Gene name } & \multicolumn{2}{|c|}{ Wild-type_Glio v Wild-type_MeOH } & \multicolumn{2}{|c|}{$\Delta g l i T \_$Glio v $\Delta g l i T \_\mathrm{MeOH}$} \\
\hline & & $\log _{2}$ (fold change) & q value & $\log _{2}$ (fold change) & $q$ value \\
\hline \multicolumn{6}{|c|}{ Siderophore biosynthesis genes } \\
\hline AFUA_1G17190 & sidl & 2.393 & 1.000 & 0.859 & 1.000 \\
\hline AFUA_1G04450 & sidL & -0.156 & 0.969 & -0.148 & 0.798 \\
\hline AFUA_1G17200 & sidC & 1.247 & 0.105 & -0.232 & 0.694 \\
\hline AFUA_2G07680 & sidA & 1.066 & 0.155 & 2.157 & 0.0005 \\
\hline AFUA_2G08590 & pptA & 0.534 & 0.738 & 0.854 & 0.143 \\
\hline AFUA_3G03400 & sidF & Present $^{\mathrm{a}}$ & 0.005 & 4.968 & 0.227 \\
\hline AFUA_3G03410 & sidH & 3.013 & 0.172 & Present $^{b}$ & 0.0005 \\
\hline AFUA_3G03420 & sidD & 4.657 & 1.000 & 2.815 & 1.000 \\
\hline AFUA_3G03650 & sidG & Present $^{a}$ & 1.000 & Present $^{b}$ & 1.000 \\
\hline AFUA_3G03660 & estB & 1.113 & 1.000 & 5.852 & 0.308 \\
\hline AFUA_5G11260 & sreA & -0.740 & 0.510 & -1.152 & 0.014 \\
\hline \multicolumn{6}{|c|}{ Siderophore-iron transport genes } \\
\hline AFUA_1G01430 & - & -1.371 & 0.080 & -2.561 & 0.0005 \\
\hline AFUA_1G14340 & - & 0.206 & 0.957 & -3.197 & 0.0005 \\
\hline AFUA_1G16040 & - & 0.081 & 0.990 & -2.115 & 0.004 \\
\hline AFUA_2G01270 & - & 0.011 & 0.997 & -1.605 & 0.031 \\
\hline AFUA_3G01360 & - & 0.251 & 0.969 & -2.778 & 0.045 \\
\hline AFUA_3G02670 & - & -1.506 & 0.054 & -2.514 & 0.0005 \\
\hline AFUA_3G03400 & sidF & Present $^{\mathrm{a}}$ & 0.005 & 4.968 & 0.227 \\
\hline AFUA_3G03440 & mirD & 2.569 & 0.005 & 3.075 & 0.0005 \\
\hline AFUA_3G13670 & - & 7.033 & 0.005 & -7.058 & 0.0005 \\
\hline AFUA_3G13700 & - & 7.619 & 0.005 & -7.824 & 0.0005 \\
\hline AFUA_4G03940 & fre7 & 2.803 & 0.005 & -2.765 & 0.005 \\
\hline AFUA_6G02170 & - & -1.026 & 0.189 & -3.820 & 0.0005 \\
\hline AFUA_6G02820 & - & 0.290 & 0.926 & -2.396 & 0.0005 \\
\hline AFUA_6G13750 & - & 2.256 & 0.005 & -0.998 & 0.146 \\
\hline AFUA_7G06060 & sit1 & 0.927 & 0.306 & 2.785 & 0.0005 \\
\hline AFUA_8G06210 & - & Present $^{a}$ & 1.000 & Absent ${ }^{c}$ & 0.0005 \\
\hline
\end{tabular}

a Switched on in ATCC46645_Glio, ${ }^{\text {b }}$ switched on in $\Delta$ gliT_Glio, ${ }^{\mathrm{C}}$ Gene expression absent in $\Delta$ gliT_Glio.

up-regulated (Table 4). In contrast, 3 genes were upregulated in $A$. fumigatus wild-type and 8 genes were down-regulated in response to exogenous gliotoxin (Table 4). In A. fumigatus $\Delta$ gliT, expression of two genes, cyp5081B1, a putative cytochrome P450 monooxygenase already mentioned in helvolic acid biosynthesis [25], and AFUA_6G00412, predicted to have amino acid transmembrane transporter activity [39] was completely abrogated in response to exogenous gliotoxin. Expression of $A$. fumigatus optB and cpsI, which are induced when BSA is the sole nitrogen source [40], was downregulated $\log _{2} 5.399$ - and $\log _{2}$ 3.98-fold, respectively, in A. fumigatus $\Delta$ gliT exposed to exogenous gliotoxin. The decreased expression of $A$. fumigatus opt $B$ in $A$. fumigatus $\Delta g l i T$ exposed to exogenous gliotoxin was confirmed by qRT-PCR (Figure 5). When utilising complex nitrogen sources, A. fumigatus secretes proteases, and increases expression of amidase, aminotransferase, and amino acid and peptide transporter genes [40,41]. These secreted proteases are regulated by a conserved regulatory factor, prtT $[40,41]$, which is decreased $\log _{2} 3.66$ fold in expression in $\Delta g l i T$ but unchanged in wild-type following exogenous gliotoxin exposure. Indeed, further examination revealed decreased expression of 14 proteases, 3 amidases and 7 amino acid and peptide transporters in $\Delta g l i T$ exposed to exogenous gliotoxin, while 
Table 4 Log $_{2}$ (fold change) in nitrogen metabolic gene, protease gene, amidase gene and animo acid transporter gene expression in $A$. fumigatus wild-type and $\Delta$ gliT exposed to exogenous gliotoxin

\begin{tabular}{|c|c|c|c|c|c|}
\hline \multirow[b]{2}{*}{ Gene } & \multirow[b]{2}{*}{ Gene name } & \multicolumn{2}{|c|}{ Wild-type_Glio v Wild-type_MeOH } & \multicolumn{2}{|c|}{$\Delta$ gliT_Glio v $\Delta$ gliT_MeOH } \\
\hline & & $\log _{2}$ (fold_change) & q_value & $\log _{2}$ (fold_change) & q_value \\
\hline AFUA_1G02780 & - & -2.005 & 0.064 & -1.839 & 0.004 \\
\hline AFUA_1G04160 & - & -1.796 & 0.015 & -3.834 & 0.0005 \\
\hline AFUA_1G04560 & - & -0.364 & 0.922 & -2.161 & 0.0005 \\
\hline AFUA_1G11250 & - & -0.610 & 0.695 & -3.225 & 0.0005 \\
\hline AFUA_1G12850 & $c r n A$ & 0.276 & 0.963 & -2.689 & 0.002 \\
\hline AFUA_1G13220 & - & 0.455 & 0.835 & -3.209 & 0.0005 \\
\hline AFUA_1G17470 & $n r t B$ & -0.098 & 0.990 & -4.504 & 0.013 \\
\hline AFUA_2G02020 & - & -0.366 & 0.962 & -4.403 & 0.0005 \\
\hline AFUA_2G02250 & - & -0.518 & 0.895 & -2.276 & 0.007 \\
\hline AFUA_2G03900 & - & -0.355 & 0.910 & -3.894 & 0.0005 \\
\hline AFUA_2G10520 & naZ & -0.780 & 0.834 & -2.793 & 0.002 \\
\hline AFUA_2G10560 & - & -0.135 & 0.988 & -4.450 & 0.0005 \\
\hline AFUA_2G12900 & - & -0.822 & 0.556 & -1.577 & 0.003 \\
\hline AFUA_2G15240 & $o p t B$ & 0.002 & 0.999 & -5.399 & 0.0005 \\
\hline AFUA_2G17430 & - & -0.680 & 0.736 & -4.444 & 0.005 \\
\hline AFUA_3G07040 & cps 1 & -0.120 & 0.984 & -3.980 & 0.0005 \\
\hline AFUA_4G01230 & - & -0.149 & 0.973 & -4.195 & 0.0005 \\
\hline AFUA_4G03770 & - & -1.008 & 0.311 & -2.329 & 0.0005 \\
\hline AFUA_4G04160 & - & -1.926 & 0.033 & -0.593 & 0.319 \\
\hline AFUA_4G04170 & - & -2.622 & 0.005 & -0.820 & 0.144 \\
\hline AFUA_4G07940 & - & -0.943 & 0.527 & -2.304 & 0.008 \\
\hline AFUA_4G14790 & сур5081B1 & 1.993 & 0.228 & Absent $^{a}$ & 0.0005 \\
\hline AFUA_5G00710 & - & 0.605 & 0.691 & -3.021 & 0.0005 \\
\hline AFUA_5G01360 & - & -0.498 & 0.891 & -3.701 & 0.024 \\
\hline AFUA_5G06230 & - & -1.355 & 0.503 & -4.916 & 0.0005 \\
\hline AFUA_5G07520 & - & -0.378 & 0.922 & -2.654 & 0.014 \\
\hline AFUA_5G13810 & - & -0.423 & 0.895 & -1.715 & 0.029 \\
\hline AFUA_5G15050 & - & -1.577 & 0.362 & -3.783 & 0.010 \\
\hline AFUA_6G00412 & - & 0.915 & 1.000 & Absent $^{a}$ & 0.0005 \\
\hline AFUA_6G01920 & - & -1.011 & 0.433 & -1.887 & 0.004 \\
\hline AFUA_6G02030 & - & -0.313 & 0.926 & -4.194 & 0.0005 \\
\hline AFUA_6G02210 & - & -1.136 & 0.161 & -3.475 & 0.0005 \\
\hline AFUA_6G05020 & - & -0.816 & 0.339 & -1.510 & 0.002 \\
\hline AFUA_6G08000 & $g m d A$ & -1.332 & 0.293 & -2.252 & 0.0005 \\
\hline AFUA_6G10210 & - & -0.664 & 0.708 & -2.153 & 0.0005 \\
\hline AFUA_7G00910 & optH & -0.132 & 0.986 & -2.089 & 0.007 \\
\hline AFUA_7G01690 & - & -1.322 & 0.269 & -1.753 & 0.0005 \\
\hline AFUA_7G02070 & $n f r 1$ & -0.453 & 0.839 & -3.250 & 0.0005 \\
\hline AFUA_7G03850 & - & -1.776 & 0.018 & -3.643 & 0.0005 \\
\hline AFUA_8G00190 & $\mathrm{ftmC}$ & -1.278 & 0.205 & -5.681 & 0.0005 \\
\hline AFUA_8G01570 & - & -1.158 & 0.561 & -3.979 & 0.003 \\
\hline AFUA_8G01780 & - & -1.171 & 0.639 & -2.758 & 0.036 \\
\hline AFUA_8G04340 & - & -0.308 & 0.910 & -1.530 & 0.001 \\
\hline
\end{tabular}


Table $4 \log _{2}$ (fold change) in nitrogen metabolic gene, protease gene, amidase gene and animo acid transporter gene expression in $A$. fumigatus wild-type and $\Delta$ gliT exposed to exogenous gliotoxin (Continued)

\begin{tabular}{|c|c|c|c|c|c|}
\hline AFUA_8G05220 & - & 0.048 & 0.996 & -2.941 & 0.0005 \\
\hline AFUA_8G06580 & - & 0.118 & 0.988 & -4.540 & 0.0005 \\
\hline AFUA_1G13440 & - & 0.520 & 0.792 & -1.692 & 0.010 \\
\hline AFUA_3G00680 & - & -4.122 & 0.005 & -1.910 & 0.0005 \\
\hline AFUA_3G14590 & - & -2.555 & 0.005 & -2.518 & 0.0005 \\
\hline AFUA_4G00630 & - & -1.078 & 0.246 & -3.158 & 0.0005 \\
\hline AFUA_4G09840 & - & -1.815 & 0.028 & -3.253 & 0.0005 \\
\hline AFUA_5G01470 & - & -0.769 & 0.739 & -4.125 & 0.001 \\
\hline AFUA_5G07360 & - & -1.768 & 0.138 & -3.948 & 0.0005 \\
\hline AFUA_7G04180 & - & -3.796 & 0.005 & -0.913 & 0.156 \\
\hline AFUA_8G01580 & - & -1.324 & 0.074 & -5.553 & 0.0005 \\
\hline AFUA_8G06470 & - & -0.077 & 0.990 & -4.663 & 0.0005 \\
\hline AFUA_5G01450 & - & -1.259 & 0.122 & -5.973 & 0.0005 \\
\hline AFUA_5G08910 & - & -1.162 & 0.102 & -3.491 & 0.0005 \\
\hline AFUA_7G06270 & - & -1.038 & 0.309 & -2.581 & 0.0005 \\
\hline AFUA_1G10820 & - & 0.846 & 0.311 & 1.730 & 0.0005 \\
\hline AFUA_2G15590 & - & 1.173 & 0.130 & 2.559 & 0.0005 \\
\hline AFUA_3G06540 & met16 & -0.276 & 0.942 & 3.029 & 0.0005 \\
\hline AFUA_6G08920 & - & -0.570 & 0.716 & 2.019 & 0.0005 \\
\hline AFUA_4G01440 & - & 2.655 & 0.018 & -1.625 & 0.251 \\
\hline AFUA_5G01662 & - & 2.626 & 0.005 & -0.025 & 0.974 \\
\hline AFUA_7G05500 & gstB & 1.991 & 0.005 & -1.228 & 0.021 \\
\hline AFUA_2G09030 & $d p p V$ & 0.411 & 0.814 & -4.442 & 0.0005 \\
\hline AFUA_4G09320 & $d p p / V$ & 1.202 & 0.347 & -6.695 & 0.0005 \\
\hline AFUA_3G00650 & lap2 & -0.065 & 0.990 & -1.608 & 0.008 \\
\hline AFUA_2G07500 & pepP & -0.790 & 0.447 & -1.933 & 0.0005 \\
\hline AFUA_4G03490 & - & -0.845 & 0.649 & -2.546 & 0.0005 \\
\hline AFUA_2G17330 & - & -0.543 & 0.707 & -1.719 & 0.001 \\
\hline AFUA_2G01250 & - & -0.683 & 0.745 & -1.989 & 0.0005 \\
\hline AFUA_6G13540 & $c p 3$ & -0.474 & 0.839 & -1.549 & 0.008 \\
\hline AFUA_6G00310 & $c p d S$ & -0.748 & 0.531 & -3.976 & 0.0005 \\
\hline AFUA_5G13300 & pep 1 & -0.833 & 0.678 & -4.357 & 0.0005 \\
\hline AFUA_3G02970 & gpra & -0.228 & 0.979 & -5.119 & 0.0005 \\
\hline AFUA_5G01200 & $c p 6$ & -0.621 & 0.834 & -4.932 & 0.0005 \\
\hline AFUA_8G00410 & metAP & -0.113 & 0.987 & -5.196 & 0.0005 \\
\hline AFUA_5G09140 & - & -0.443 & 0.910 & 3.372 & 0.002 \\
\hline AFUA_5G02990 & - & -0.805 & 0.512 & -4.047 & 0.0005 \\
\hline AFUA_1G09120 & - & -0.272 & 0.962 & -2.985 & 0.002 \\
\hline AFUA_8G00800 & - & 0.641 & 0.899 & -6.795 & 0.0005 \\
\hline AFUA_1G12240 & - & 0.347 & 0.964 & -5.161 & 0.0005 \\
\hline AFUA_8G02550 & - & 0.564 & 0.819 & -8.305 & 0.003 \\
\hline AFUA_7G04290 & - & -0.464 & 0.910 & -2.355 & 0.0005 \\
\hline AFUA_8G05860 & - & -0.463 & 0.861 & -2.099 & 0.003 \\
\hline
\end{tabular}

${ }^{\mathrm{a} G e n e ~ e x p r e s s i o n ~ a b s e n t ~ i n ~} \Delta g$ lit_Glio. 
expression of one amidase, AFUA_5G09140, was increased $\log _{2}$ 3.37-fold (Table 4). Expression of both nitrate transporters, $c r n A$ and $n r t B$, which are predicted gene pairs [42], was significantly down-regulated ( $\log _{2}$ 2.689- and $\log _{2}$ 4.504-fold) in A. fumigatus $\Delta g l i T$ upon exogenous gliotoxin exposure. Expression of a number of genes with predicted involvement in the oxidationreduction process was down-regulated in A. fumigatus $\Delta g l i T$ only following exogenous gliotoxin exposure. Among these genes were nitronate monooxygenases (AFUA_2G17430 and AFUA_4G07940 down-regulated $\log _{2}$ 4.444- and $\log _{2}$ 2.304-fold) and copper ion binding domain genes (AFUA_1G13440, AFUA_5G01470 and AFUA_5G07360; down-regulated $\log _{2} 1.692-, \log _{2} 4.125$ - and $\log _{2}$ 3.948-fold, respectively). In $A$. fumigatus wild-type exposed to exogenous gliotoxin, two predicted carbon-nitrogen ligases, AFUA_4G04160 and AFUA_4G04170, were downregulated $\log _{2} 1.926$ - and $\log _{2}$ 2.622-fold, respectively. These carbon-nitrogen ligases are not differentially regulated in A. fumigatus $\Delta g l i T$ upon exogenous gliotoxin exposure, suggesting that loss of gliT may hinder the downregulation of these genes in response to gliotoxin.

\section{Exogenous gliotoxin exposure alters gene expression involved in ribosome biogenesis and translation in $A$. fumigatus $\Delta$ gliT while transcription related genes are down-regulated}

Dysregulation of ribosome biogenesis and translation was observed in A. fumigatus $\Delta$ gliT following exogenous gliotoxin exposure, whereby expression of 20 genes was up-regulated and two genes down-regulated (Table 5). Of the 20 genes up-regulated in A. fumigatus $\Delta g l i T$ in response to exogenous gliotoxin, 7 genes encode $60 \mathrm{~S}$ ribosomal proteins and two encode $40 \mathrm{~S}$ ribosomal proteins, all of which are increased $\log _{2} 1.5$-1.7-fold in expression (Table 5). The two genes down-regulated in response to exogenous gliotoxin in A. fumigatus $\Delta$ gliT have already been mentioned as secondary metabolite biosynthetic genes. A. fumigatus metAP in the fumagillin biosynthetic gene cluster [30] was down-regulated $\log _{2}$ 4.340-fold and A. fumigatus elfB in the pseurotin A biosynthetic gene cluster was down-regulated $\log _{2} 7.265$ fold $[27,31]$. In contrast to A. fumigatus $\Delta g l i T$, only two genes involved in ribosome biogenesis and translation were up-regulated in A. fumigatus wild-type exposed to exogenous gliotoxin (Table 5). AFUA_5G07340 was up-regulated $\log _{2}$ 1.909-fold while AFUA_2G12150 was up-regulated $\log _{2} 2.417$-fold. In addition to the dysregulation of ribosome biogenesis and translation, 44 genes involved in transcription were also down-regulated in A. fumigatus $\Delta g l i T$ in response to exogenous gliotoxin, compared to 3 down-regulated genes in wild-type exposed to exogenous gliotoxin (Table 6). A proteomic investigation of $A$. fumigatus $\triangle$ gliT $T^{A T C C 26933}$ reflected this alteration in translation whereby 6 proteins were uniquely expressed or increased in abundance and 15 proteins were absent or decreased in abundance when exposed to exogenous gliotoxin (Tables 7 and 8). Loss of GliT negatively impacts expression of genes involved in transcription processes and results in significantly decreased transcription of genes in the presence of exogenous gliotoxin, culminating in disruption of translation.

\section{Discussion}

To dissect the role played by the gliotoxin oxidoreductase, gliT, in self-protection against gliotoxin, a comparative transcriptomic analysis of the impact of exogenous gliotoxin on A. fumigatus wild-type and $\Delta g l i T$ was carried out via RNA-seq analysis. To reduce the affects of endogenous gliotoxin, a low gliotoxin-producing background strain, $A$. fumigatus ATCC46645 [2] was employed, along with Sabouraud-Dextrose media which is non-permissive for gliotoxin biosynthesis [5]. Exogenous gliotoxin exposure resulted in changed expression of 164 genes in A. fumigatus wild-type. However, altered expression of over 1700 genes was observed in $A$. fumigatus $\Delta g l i T$. Closer inspection revealed alterations in expression of clusters encoding secondary metabolites, particularly gliotoxin, helvolic acid, fumitremorgin, fumagillin and pseurotin A biosynthesis genes, siderophore biosynthesis genes, ribosome biogenesis genes and genes involved in translation and nitrogen metabolism.

Exogenous gliotoxin induces the expression of gliotoxin biosynthetic genes in both $A$. fumigatus wild-type and $\Delta g l i T$. Expression of gliZ was increased in expression in wild-type, along with gliP, the bimodular non-ribosomal peptide synthetase that produces the cyclo-L-Phe-L-Ser diketopiperazine intermediate $[9,10,43]$. Up-regulation of gliZ and gliP expression in particular, along with gliF suggests that exogenous gliotoxin induces de novo gliotoxin production in wild-type. Indeed this has been shown to be the case in A. fumigatus Af293 where addition of exogenous gliotoxin under gliotoxin-inducing culture conditions resulted in detection of de novo gliotoxin production determined by the use of $\left[{ }^{13} \mathrm{C}\right]$-Phenylalanine and LC-MS analysis. Recently, cyclo-L-Phe-L-Ser was determined to be a major component of the metabolome and was detected in lung tissue of infected mice $[11,44]$. The production of this compound may be increased consequent to exogenous gliotoxin exposure in wild-type due to upregulation of gliP expression. It has been demonstrated that $A$. fumigatus gliT expression is not solely under the control of gliZ [2], and so up-regulation of gliZ expression is not absolutely required to induce gliT expression for the purpose of self-protection. While gliZ expression is not significantly altered in $\Delta g l i T$ following exogenous gliotoxin exposure in the RNA-seq analysis, qRT-PCR demonstrated increased expression under these conditions. 
Table 5 Log $_{2}$ (fold change) in ribosome biogenesis and translation gene expression in A. fumigatus wild-type and $\Delta$ gliT exposed to exogenous gliotoxin

\begin{tabular}{|c|c|c|c|c|c|}
\hline \multirow[b]{2}{*}{ Gene } & \multirow[b]{2}{*}{ Gene name } & \multicolumn{2}{|c|}{ Wild-type_Glio v Wild-type_MeOH } & \multicolumn{2}{|c|}{$\Delta$ gliT_Glio v $\Delta$ gliT_MeOH } \\
\hline & & $\log _{2}$ (fold_change) & q_value & $\log _{2}$ (fold_change) & q_value \\
\hline AFUA_3G05600 & - & 1.122 & 0.228 & 1.647 & 0.004 \\
\hline AFUA_3G06760 & - & 1.249 & 0.075 & 1.763 & 0.001 \\
\hline AFUA_5G07340 & - & 1.909 & 0.012 & 0.268 & 0.728 \\
\hline AFUA_6G05200 & - & 1.061 & 0.227 & 1.590 & 0.008 \\
\hline AFUA_6G13250 & - & 1.203 & 0.117 & 1.609 & 0.003 \\
\hline AFUA_8G00410 & metAP & -0.113 & 0.987 & -4.340 & 0.001 \\
\hline AFUA_8G00580 & elfB & 0.306 & 0.964 & -7.265 & 0.003 \\
\hline AFUA_2G09200 & - & 1.100 & 0.143 & 1.510 & 0.003 \\
\hline AFUA_3G07360 & - & 0.492 & 0.739 & 1.506 & 0.004 \\
\hline AFUA_1G12890 & - & 1.191 & 0.212 & 1.595 & 0.006 \\
\hline AFUA_1G15020 & - & 1.036 & 0.277 & 1.545 & 0.008 \\
\hline AFUA_2G10300 & - & 1.353 & 0.060 & 1.537 & 0.004 \\
\hline AFUA_2G12150 & - & 2.417 & 0.005 & 0.561 & 0.422 \\
\hline AFUA_3G08080 & - & 0.214 & 0.952 & 1.655 & 0.001 \\
\hline AFUA_4G07250 & - & 0.599 & 0.589 & 1.572 & 0.002 \\
\hline AFUA_4G07730 & - & 1.190 & 0.163 & 1.592 & 0.004 \\
\hline AFUA_4G11990 & - & 0.309 & 0.891 & 1.682 & 0.001 \\
\hline AFUA_5G05630 & - & 1.222 & 0.122 & 1.661 & 0.002 \\
\hline AFUA_5G06430 & - & 0.559 & 0.649 & 1.501 & 0.002 \\
\hline AFUA_6G02440 & - & 1.006 & 0.272 & 1.658 & 0.002 \\
\hline AFUA_6G02450 & - & 0.714 & 0.493 & 1.564 & 0.003 \\
\hline AFUA_6G11260 & - & 1.105 & 0.232 & 1.532 & 0.008 \\
\hline AFUA_6G12960 & - & 0.521 & 0.686 & 1.721 & 0.001 \\
\hline AFUA_2G10100 & aspf8 & 1.295 & 0.138 & 1.580 & 0.007 \\
\hline
\end{tabular}

Expression of a number of gli genes was up-regulated in $\Delta g l i T$ following exogenous gliotoxin exposure, including gliM, which was not altered in wild-type. A. fumigatus gliM is a predicted $O$-methyltransferase [3] and methylation has been proposed as a method of self-protection against disulfide bridge-containing metabolites. Holomycin contains a disulfide bridge similar to gliotoxin $[16,17,45]$ and $S$-methylation has been proposed as an alternative method of self-protection against this antibiotic and its biosynthetic intermediates in S. clavuligerus upon deletion of the dithiol oxidase, HlmI, which is functionally homologous to A. fumigatus gliT [17,45]. In Y. ruckeri, an RNA methyltransferase, Hom12, methylates RNA in a proposed self-protection mechanism against the cytotoxic effects of holomycin [16]. Interestingly, in A. fumigatus $\Delta g l i T$ following exogenous gliotoxin exposure, a tRNA methyltransferase, AFUA_4G12280, is up-regulated $\log _{2} 2.56$ fold, while its expression is unaltered in wild-type in the presence of exogenous gliotoxin. The increased expression of these methyltransferases, among others, in A. fumigatus
$\Delta g l i T$ following exposure to exogenous gliotoxin may suggest possible alternative functionalities, or selfprotection mechanisms against gliotoxin in the absence of GliT. Interestingly, a newly-identified methyltransferase, gliotoxin methyltransferase A (GtmA), has been demonstrated to play a role in attenuating gliotoxin biosynthesis [46]. GtmA (AFUA_2G11120) expression is significantly up-regulated $(p<0.00005)$ by gliotoxin exposure (Additional file 1).

In addition to up-regulation of the gli cluster, dysregulation of other secondary metabolite gene clusters was observed upon exogenous gliotoxin addition, particularly to A. fumigatus $\Delta$ gliT. Up-regulated expression of two genes; osc3 and sdrI, from the gene cluster encoding the fusidane antibiotic helvolic acid was observed upon exogenous gliotoxin addition to A. fumigatus wild-type [25]. However, exogenous gliotoxin addition to A. fumigatus $\Delta g l i T$ resulted in down-regulated expression of two genes and abrogation of the expression of cyp5081D1, suggesting that gliT deletion, along with exogenous gliotoxin exposure 
Table $6 \log _{2}$ (fold change) in transcription gene expression in A. fumigatus wild-type and $\Delta$ gliT exposed to exogenous gliotoxin

\begin{tabular}{|c|c|c|c|c|c|}
\hline \multirow[b]{2}{*}{ Gene } & \multirow[b]{2}{*}{ Gene name } & \multicolumn{2}{|c|}{ Wild-type_Glio v Wild-type_MeOH } & \multicolumn{2}{|c|}{$\Delta$ gliT_Glio v $\Delta$ gliT_MeOH } \\
\hline & & $\log _{2}$ (fold_change) & q_value & $\log _{2}$ (fold_change) & q_value \\
\hline AFUA_4G11480 & - & -0.766 & 0.446 & -1.532 & 0.003 \\
\hline AFUA_6G05160 & $a z f 1$ & -1.541 & 0.043 & -0.251 & 0.753 \\
\hline AFUA_6G12150 & - & 0.502 & 0.913 & -6.233 & 0.001 \\
\hline AFUA_7G01340 & - & 0.082 & 0.990 & -2.982 & 0.001 \\
\hline AFUA_4G02930 & - & 0.450 & 0.796 & -1.971 & 0.001 \\
\hline AFUA_3G10120 & - & -0.819 & 0.488 & -1.669 & 0.003 \\
\hline AFUA_4G10110 & $h t f A$ & 0.105 & 0.986 & -1.565 & 0.012 \\
\hline AFUA_1G10080 & zafA & -0.434 & 0.843 & -1.535 & 0.001 \\
\hline AFUA_1G01240 & - & -0.561 & 0.770 & -1.702 & 0.025 \\
\hline AFUA_1G14945 & - & -0.839 & 0.505 & -1.754 & 0.026 \\
\hline AFUA_2G04262 & - & -1.644 & 0.012 & -0.759 & 0.171 \\
\hline AFUA_2G11180 & $f l b A$ & -0.126 & 0.977 & -1.511 & 0.003 \\
\hline AFUA_3G03330 & - & -2.132 & 0.021 & -0.739 & 0.221 \\
\hline AFUA_5G03780 & - & 1.277 & 0.320 & -2.864 & 0.001 \\
\hline AFUA_5G09720 & - & -1.780 & 0.086 & -1.586 & 0.033 \\
\hline AFUA_5G14530 & - & 0.120 & 0.987 & -2.166 & 0.010 \\
\hline AFUA_8G01150 & - & 0.037 & 0.997 & -3.345 & 0.001 \\
\hline AFUA_8G01940 & - & -1.248 & 0.221 & -1.846 & 0.027 \\
\hline AFUA_8G02720 & - & -0.032 & 0.997 & -1.848 & 0.001 \\
\hline AFUA_8G04130 & farB1 & -0.496 & 0.798 & -1.898 & 0.001 \\
\hline AFUA_8G06460 & - & -0.005 & 0.998 & -1.741 & 0.009 \\
\hline AFUA_6G11740 & - & -0.919 & 0.475 & -7.145 & 0.004 \\
\hline AFUA_2G05180 & - & -0.361 & 0.891 & -3.819 & 0.001 \\
\hline AFUA_1G16600 & - & -0.343 & 0.964 & -4.481 & 0.001 \\
\hline AFUA_8G00280 & - & -0.720 & 0.574 & -1.813 & 0.007 \\
\hline
\end{tabular}

negatively regulates the helvolic acid gene cluster. In an A. fumigatus gliZ complemented strain, helvolic acid production was detectable at $37^{\circ} \mathrm{C}$, whereas no helvolic acid was detectable in wild-type or $\Delta g l i Z$ strains at an identical growth temperature [10], suggesting that gliZ may be

Table 7 Proteins with increased abundance in, or unique to, A. fumigatus $\Delta$ glit $^{\mathrm{ATCC} 26933}$ with gliotoxin compared to the methanol control

\begin{tabular}{ccccc}
\hline Protein IDs & $\begin{array}{c}\log _{2} \text { (fold } \\
\text { increase) }\end{array}$ & $\boldsymbol{p}$ value & Peptides & $\begin{array}{c}\text { Sequence } \\
\text { coverage [\%] }\end{array}$ \\
\hline AFUA_6G06470 & Unique & N/A & 6 & 58.5 \\
AFUA_1G09510 & Unique & N/A & 2 & 34.5 \\
AFUA_4G03140 & Unique & N/A & 4 & 12.5 \\
AFUA_3G14540 & 2.279 & $1.52 E-02$ & 6 & 38.3 \\
AFUA_1G15270 & 1.135 & 2.05E-02 & 33 & 43.1 \\
AFUA_7G00350* & 1.119 & 1.49E-02 & 6 & 23.2 \\
\hline
\end{tabular}

*Gene expression also significantly up-regulated in RNA-seq analysis. involved in regulating helvolic acid production. Indeed in the present study in wild-type, gliZ expression was upregulated upon gliotoxin exposure and the helvolic acid biosynthetic genes, including osc 3 which encodes the protein that catalyses the first biosynthetic step of helvolic acid biosynthesis [25], are also up-regulated, whereas in $\Delta g l i T$, the helvolic acid biosynthesis genes are downregulated. However, we cannot unambiguously assign gliZ functionality to hevolic acid production because consistently discrepant $g l i Z$ expression data was obtained by RNA-seq and qRT-PCR (Figure 5).

The combined loss of gliT and exposure to exogenous gliotoxin resulted in attenuated expression of the biosynthetic genes for the secondary metabolites: fumitremorgin B, fumagillin, and pseurotin A. Secondary metabolite production in fungi is generally dependant on the presence of a key backbone enzyme, namely a PKS or a NRPS [47]. In A. fumigatus $\Delta g l i T$ exposed to exogenous gliotoxin, the NRPS ftmA in the fumitremorgin B cluster, the PKS, fma- 
Table 8 Proteins with decreased abundance in, or absent from, $\Delta$ gliT $^{\mathrm{ATCC} 26933}$ with gliotoxin compared to the methanol control

\begin{tabular}{ccccc}
\hline Protein IDs & $\begin{array}{c}\text { Log }_{2} \text { (fold } \\
\text { decrease) }\end{array}$ & $\boldsymbol{p}$ value & Peptides & $\begin{array}{c}\text { Sequence } \\
\text { coverage [\%] }\end{array}$ \\
\hline AFUA_3G00330* & Unique & N/A & 6 & 36.2 \\
AFUA_5G14000* & Unique & N/A & 7 & 61.3 \\
AFUA_1G10960 & Unique & N/A & 2 & 18.2 \\
AFUA_8G00540* & 3.032 & $1.72 E-02$ & 37 & 18.7 \\
AFUA_2G15290 & 2.788 & $6.36 \mathrm{E}-04$ & 6 & 81.6 \\
AFUA_8G00440* & 2.461 & $7.89 \mathrm{E}-04$ & 12 & 21.2 \\
AFUA_7G06420 & 2.451 & $3.18 \mathrm{E}-03$ & 18 & 63.5 \\
AFUA_8G00550* & 2.016 & $1.03 \mathrm{E}-02$ & 12 & 63.8 \\
AFUA_2G04060* & 1.799 & $5.53 \mathrm{E}-03$ & 12 & 58.7 \\
AFUA_3G03350* & 1.603 & $2.31 \mathrm{E}-03$ & 50 & 38.6 \\
AFUA_5G07170* & 1.361 & $3.93 \mathrm{E}-02$ & 7 & 44.9 \\
AFUB_044910 & 1.234 & $1.02 \mathrm{E}-02$ & 16 & 61.3 \\
AFUA_1G01010 & 1.128 & $4.74 \mathrm{E}-02$ & 33 & 26.4 \\
AFUA_8G05580 & 1.091 & $1.12 \mathrm{E}-02$ & 11 & 43.2 \\
AFUA_6G10120 & 1.072 & $2.60 \mathrm{E}-02$ & 9 & 46.4 \\
\hline
\end{tabular}

*Gene expression also significantly down-regulated in RNA-seq analysis.

PKS in the fumagillin cluster and the PKS-NRPS hybrid psoA/nrps14 in the pseurotin A cluster were all significantly down-regulated suggesting production of these secondary metabolites is also decreased. A. fumigatus ftmA encodes the enzyme required for the first biosynthetic step of fumitremorgin synthesis, the synthesis of the diketopiperazine, brevianamide $\mathrm{F}$ [29], and is significantly downregulated $\log _{2}$ 6.640-fold in A. fumigatus $\Delta$ gliT following exogenous gliotoxin addition. Expression of the cytochrome $\mathrm{P} 450 \mathrm{~A}$. fumigatus ftmE, which was absent in $\Delta g l i T$ exposed to exogenous gliotoxin, encodes the enzyme responsible for formation of fumitremorgin $C$ [48], while another cytochrome $\mathrm{P} 450$, ftmG, which encodes the enzyme that dihydroxylates the fumitremorgin $\mathrm{B}$ intermediate was significantly down-regulated $\log _{2} 6.519$-fold [48]. We hypothesise that fumitremorgin $\mathrm{B}$ biosynthesis is decreased, if not abrogated, in A. fumigatus $\Delta$ gliT when exposed to exogenous gliotoxin given the decreased expression of genes essential for synthesis. While determination of fumitremorgin B levels was not successful, measurement of fumitremorgin $\mathrm{C}$ and related compounds [48] was carried out in A. fumigatus wild-type and $\Delta g l i T$ cultured under secondary metabolite-inducing conditions. Significant reductions in the levels of tryprostatin A and tryprostatin B were observed in A. fumigatus $\Delta g l i T$ compared to wild-type, while there was no significant difference in levels of fumitremorgin $C$ between the two strains. Therefore, GliT loss disrupts the production of brevianamide $\mathrm{F}$, tryprostatin $\mathrm{A}$ and tryprostatin $\mathrm{B}$, from the fumitremorgin biosynthetic pathway in A. fumigatus. The PKS, fma-PKS, down-regulated $\log _{2} 7.287$-fold in $A$. fumigatus $\Delta g l i T$, is essential for fumagillin biosynthesis [30]. Additionally, the C6 type transcription factor fapR/ fumR, which controls the expression of the other fumagillin cluster genes [22], was down-regulated $\log _{2} 5.826$-fold in A. fumigatus $\Delta g l i T$ following exogenous gliotoxin addition. Decreased expression of these two genes, in addition to others in the biosynthetic gene cluster, suggests down-regulation of fumagillin biosynthesis as a direct consequence of $A$. fumigatus gliT loss combined with exogenous gliotoxin stress. Indeed supporting the hypothesis of A. fumigatus gliT involvement in facilitating fumagillin biosynthesis, under secondary metabolite-inducing conditions we observed a significant decrease in the production of fumagillin in A. fumigatus $\Delta g l i T$ compared to wild-type. It has been determined that the biosynthetic genes for fumagillin and pseurotin A are physically intertwined [27] and these authors also revealed that fapR, which controls expression of the fumagillin biosynthesis genes, also controls the expression of pseurotin A biosynthesis genes [27]. Indeed, with the exception of one gene, expression of the pseurotin A biosynthetic cluster was significantly down-regulated in A. fumigatus $\Delta g l i T$ upon exogenous gliotoxin addition. psoA/nrps14 is essential for pseurotin A biosynthesis, as demonstrated by Maiya et al. [28], while over-expression of this gene increased pseurotin A accumulation. In $A$. fumigatus $\Delta g l i T$ exposed to exogenous gliotoxin, psoA/nrps14 expression was downregulated $\log _{2} 5.826$-fold, while in the proteomic investigation, PsoA/nrps14 was decreased $\log _{2}$ 3.032-fold in abundance. In addition to PsoA/nrps14, two other proteins required for pseurotin A biosynthesis [27] were decreased in abundance upon exogenous gliotoxin exposure in A. fumigatus $\Delta g l i T$. PsoF (AFUA_8G00440) and PsoC (AFUA_8G00550) were decreased $\log _{2} 2.461$ - and $\log _{2}$ 2.016-fold providing further support to the hypothesis that pseurotin A biosynthesis is down-regulated in the absence of A. fumigatus gliT when challenged with exogenous gliotoxin. As was the case with fumagillin, pseurotin A levels were significantly decreased in A. fumigatus $\Delta g l i T$ compared to wild-type when cultured under secondary metabolite-inducing conditions again suggesting possible GliT involvement in enabling pseurotin A biosynthesis.

The biosynthetic gene clusters of fumitremorgin, fumagillin and pseurotin are under the control of the global regulator, A. fumigatus laeA [26,27]. A. fumigatus lae $A$ is a methyltransferase and regulates chromatin remodelling through this methyltransferase activity $[26,32,49]$. In $A$. fumigatus $\Delta g l i T$ treated with exogenous gliotoxin, laeA expression was not significantly altered, suggesting that it is not responsible for the downregulation of these gene clusters. In $A$. nidulans, LaeA forms a trimeric complex with two members of the 
velvet protein family; VeA and VelB, and this complex up-regulates secondary metabolism and sexual development [50]. The complex was subsequently identified and characterised in A. fumigatus [34]. A. fumigatus veA has been demonstrated to regulate fumagillin and gliotoxin production [20,22], however veA expression was not significantly altered in $A$. fumigatus $\triangle g l i T$ following exogenous gliotoxin production. The bZip transcriptional enhancer RsmA has been shown to positively regulate gliotoxin biosynthesis [44], however the expression of A. fumigatus rsmA was not altered significantly in A. fumigatus $\triangle g l i T$, in the absence or presence of exogenous gliotoxin. Overall, our findings lead us to postulate that the gliotoxin biosynthetic/self-protection capacity (i.e., GliT functionality) is necessary for optimal biosynthesis of selected secondary metabolites in A. fumigatus.

Altered expression of siderophore-iron transport and siderophore biosynthetic genes in both A. fumigatus wild-type and $\Delta g l i T$ following exogenous gliotoxin exposure suggests a disruption in iron homeostasis or iron sensing. Iron is an essential nutrient that is required for many cellular processes, including as a cofactor for numerous enzymes [35]. In both wild-type and $\Delta g l i T$, siderophore biosynthesis gene expression was up-regulated in response to exogenous gliotoxin indicating that consequent to exogenous gliotoxin exposure, there is an increased requirement for iron despite sufficient iron availability in the culture media, or else a deficit in ironsensing. A. fumigatus sidF expression was activated in wild-type, while in $\Delta g l i T$, sidH expression was activated and that of $\operatorname{sid} A$ was up-regulated [35]. Interestingly, upregulation of sidA expression has also been observed in $\Delta m e t R$, an A. fumigatus mutant deficient in the transcription factor that regulates sulfur assimilation, under iron sufficient but sulfur deficient conditions [21]. Regulatory cross-talk between secondary metabolism and iron requirement has been reported whereby in a laeA mutant deficient in gliotoxin production, decreased expression of a number of the siderophore biosynthesis genes was observed under high iron conditions [26]. These authors concluded that laeA was also involved in regulating expression of the siderophore biosynthetic genes and in particular, sidD. As discussed, we have observed that the combined effect of exogenous gliotoxin exposure and gliT deletion has a significant impact on secondary metabolism, despite laeA expression being unaffected, and therefore it is interesting that siderophore biosynthesis is also affected. In A. fumigatus wild-type, expression of six siderophore-iron transport genes was up-regulated in response to exogenous gliotoxin, in contrast to up-regulation of two siderophore-iron transport genes in $\Delta g l i T$. Interestingly, in $\Delta g l i T$, expression of 12 siderophore-iron transport genes was down-regulated. Taken together, this suggests that deletion of gliT in combination with exogenous gliotoxin exposure results in a disruption of, or decrease in, siderophore-iron transport. Amich et al. [21] noted up-regulation of siderophore-iron transport genes in $\Delta m e t R$ under sulfur-limited but iron replete conditions. Despite observing increased expression of both siderophore biosynthetic genes and siderophoreiron transport genes in $\Delta m e t R$, suggesting iron starvation, the authors noted increased levels of ferricrocin, the intracellular siderophore that is used for transport and storage [38,51]. Iron is utilised in many processes one of which is iron-sulfur cluster biosynthesis. Iron-sulfur clusters are inorganic cofactors involved in cellular processes including enzyme activity regulation, mitochondrial respiration, ribosome biosynthesis and cofactor biosynthesis [52]. Translocation of iron-sulfur clusters requires glutathione, and depletion has been shown to induce an iron starvation-like response in Saccharomyces cerevisiae [53-55]. It has previously been shown that exogenous gliotoxin exposure to both $A$. fumigatus wild-type and $\Delta g l i T$ results in decreased GSH levels [5] and so it is conceivable that this decrease in cellular GSH could impact on iron homeostasis or iron sensing.

A. fumigatus encodes two transcription factors, hapX and $s r e A$, that maintain iron homeostasis whereby hapX represses sreA expression and subsequently ironconsuming pathways, and activates siderophore biosynthesis during iron-starvation, while sreA represses hapX during iron-sufficient conditions in a negative feedback loop [35,56]. Interestingly, induction of gliT has been shown before when cultures were shifted from ironlimited to replete conditions in both wild-type and a sreA deletion strain [57]. Although expression of hapX and $s r e A$ is unchanged in both wild-type and $\Delta g l i T$ upon exogenous gliotoxin exposure, there is significant interplay between gliT, sulfur and iron as demonstrated by the altered expression of siderophore biosynthesis and siderophore-iron transport genes and the decreased GSH levels [5] in A. fumigatus $\Delta g l i T$.

FunCat analysis identified significant enrichment of genes involved in nitrogen metabolism in the downregulated gene set in $\Delta g l i T$ exposed to exogenous gliotoxin. Fungi can utilise various sources of nitrogen, from easily assimilated sources (e.g., ammonium and glutamate), to more complex secondary sources including amino acids and proteins $[40,58]$. Following exposure to exogenous gliotoxin, the expression of $\operatorname{prt} T$, the conserved regulator of secreted proteases, was decreased and consequentially, a number of proteases previously identified to be under its control were also decreased in expression, including alp1 and pep1 [41]. Additionally, a number of other proteases underwent decreased expression in $\Delta g l i T$ when exposed to exogenous gliotoxin. These proteases, some of which include $d p p V, d p p V I, c p d S$ and $g p r A$, have been shown to be induced when BSA is the sole nitrogen source [41]. In 
order to conserve energy, fungi will preferentially utilise nitrogen sources that are easily assimilated over complex nitrogen sources [59]. Sabouraud-Dextrose medium contains a pancreatic digest of casein and a peptic digest of animal tissue. As the media already contained digested proteins, we conclude that expression of these secreted proteases is significantly down-regulated in $\Delta g l i T$ exposed to exogenous gliotoxin in order to conserve energy. Interestingly, a number of genes involved in amino acid and peptide transport are also decreased in expression with the exception of one amidase, AFUA_5G09140, the expression of which is up-regulated in $\Delta g l i T$ following exogenous gliotoxin exposure. This was surprising as, despite the availability of amino acids and peptides in the media, the expression of genes encoding enzymes required for uptake of these amino acids and peptides are down-regulated. It is possible that sufficient enzymes are present for adequate uptake of nitrogen sources to compensate for the decreased expression of these genes, or that the down-regulation of these genes is a consequence of the down-regulation of $\operatorname{prt} T$ and the other secreted proteases suggesting that there is a decreased nitrogen requirement in $\Delta g l i T$, when exposed to exogenous gliotoxin.

Loss of $A$. fumigatus gliT had a significant impact on the transcriptome when challenged with exogenous gliotoxin whereby expression of 1,700 genes was altered, of which 1192 were down-regulated, that was not observed in wild-type where 164 genes had altered expression. This suggests that consequent to GliT absence, transcription is suppressed in the presence of exogenous gliotoxin. This is further supported by the decreased expression of 44 genes involved in transcription processes, many of which are transcription factors. Further to the effects of gliT deletion on transcription, translation is also disrupted in A. fumigatus $\Delta g l i T$ following exposure to exogenous gliotoxin. Altered expression of genes required for ribosome biogenesis and translation processes was observed in $\Delta g l i T$. To support the hypothesis that translation is disrupted, the LFQ proteomic investigation of $A$. fumigatus $\Delta$ gliT did not reflect the large transcriptome changes, as only 6 proteins with increased abundance or uniquely present, and 15 proteins absent or with decreased abundance were identified. It is important to note that despite the low number of proteins altered in abundance, there is agreement between the RNA-seq analysis and the proteomic analysis of A. fumigatus $\Delta g l i T$ exposed to exogenous gliotoxin, whereby the genes encoding one of the proteins increased in abundance and 8 of the proteins decreased in abundance were up-regulated and down-regulated, respectively.

\section{Conclusions}

We present the first global investigation of the transcriptional response to exogenous gliotoxin in A. fumigatus wild-type and $\Delta g l i T$ employing RNA-seq analysis. While exogenous gliotoxin elicits some transcriptome remodelling in wild-type, in $\Delta g l i T$, the transcriptional response is 10 -fold that of wild-type, with approximately $70 \%$ of these altered genes decreased in expression. We found that the combined loss of gliT and exogenous gliotoxin exposure results in decreased expression of a number of secondary metabolite genes from the biosynthetic clusters of helvolic acid, fumitremorgin $\mathrm{B}$, pseurotin $\mathrm{A}$ and fumagillin despite the unchanged expression of laeA, the global regulator of secondary metabolism. Thus, GliT functionality may extend to enhancing the biosynthesis of selected secondary metabolites in $A$. fumigatus. In addition to this, the decreased expression of many transcription factor genes, along with genes involved in siderophore-iron transport and siderophore biosynthesis and nitrogen metabolism indicates that exogenous gliotoxin induces a starvation-like response despite the use of a rich media. Furthermore, the combined RNA-seq and proteomic analysis revealed deletion of gliT abrogated transcription and disrupts translation when exposed to exogenous gliotoxin. Overall, this study provides a detailed overview of the response to exogenous gliotoxin in resistant and sensitive A. fumigatus strains, enhances our understanding of the manner in which gliotoxin exerts its affects as a toxin, and provides a unique glimpse into cross-talk between apparently unrelated secondary metabolite gene clusters.

\section{Methods \\ Aspergillus fumigatus strain information and growth conditions}

Conidia were maintained on malt extract agar plates. $A$. fumigatus wild-type and mutant strains $\left(1 \times 10^{6} \mathrm{cfu} / \mathrm{ml}\right)$ were cultured in Sabouraud-Dextrose media at $37^{\circ} \mathrm{C}$ with shaking at $200 \mathrm{rpm}$ for $21 \mathrm{~h}$ in duplicate or triplicate. Gliotoxin $(5 \mu \mathrm{g} / \mathrm{ml}$ final) or $\mathrm{MeOH}$ (solvent control) was added and the cultures were incubated for a further $3 \mathrm{~h}$. The mycelia were harvested through miracloth, washed with DEPC water and snap frozen in liquid $\mathrm{N}_{2}$.

\section{RNA extraction and mRNA isolation}

RNA was isolated from mycelia, ground to a fine powder in liquid $\mathrm{N}_{2}$, using the RNeasy ${ }^{\mathrm{TM}}$ Plant Mini Kit (Qiagen), according to the manufacturer's instructions. RNA integrity was analysed using an Agilent 2100 Bioanalyzer $^{\text {тM }}$ and an Agilent RNA 6000 Nano Kit following the manufacturer's recommendation.

\section{Library preparation and sequencing}

A library was independently prepared for each biological replicate. Two protocols (TruSeq and Illumina mRNA-seq kit) were used for preparing the Illumina transcriptome libraries. For both protocols, polyadenylated mRNA was 
purified from total RNA using oligo-dT dynabead selection followed by metal ion hydrolysis fragmentation with an RNA fragmentation solution supplied in kits. First strand synthesis, primed using random oligonucleotides, was followed by second strand synthesis with $\mathrm{RNaseH}$ and DNA polI to produce double-stranded cDNA using the Illumina mRNA Seq kit or the TruSeq Illumina kit. Template DNA fragments were end-repaired with T4 and Klenow DNA polymerases and blunt-ended with T4 polynucleotide kinase. A single 3' adenosine was added to the repaired ends using Klenow exo- and dATP to reduce template concatemerization and adapter dimer formation, and to increase the efficiency of adapter ligation. Adapters (containing primer sites for sequencing, and index sequences when using the TruSeq protocol) were then ligated. Libraries made with the TruSeq protocol were amplified by PCR using KAPA HiFi Polymerase (to enrich for properly ligated template strands, to generate enough DNA, and to add primers for flowcell surface annealing). AMPure SPRI beads were used to purify amplified templates before pooling based on quantification using an Agilent Bioanalyser chip. Pooled TruSeq libraries were then pooled and size selected using the Caliper. After adaptor ligation, individual libraries made with the Illumina mRNA-seq kit were size selected using the CaliperLabChip before PCR amplification followed by AMPure SPRI bead clean up and removal of adaptors with a second Caliper run. KAPA Illumina SYBR Fast qPCR kit was used to quantify the Illumina mRNA-seq libraries before pooling. No qPCR was necessary with the TruSeq libraries and instead a final Agilent Bioanalyser chip analysis was run to confirm the dilution of the final pool. The libraries were sequenced on the Illumina HiSeq platform with a read length of 75 bp paired-ended according to manufacturer's instructions.

\section{Data processing}

The RNA-seq paired-end reads for each biological replicate were aligned independently using Tophat v2.0.4 [23618408] to the A. fumigatus Af293 (CADRE 3a) reference genome sequence with default parameters. The numbers of fragments mapped per replicate is given in Additional file 2: Table S13. Differential gene expression analysis was carried out for each sample independently using Cufflinks (cuffdiff) v2.0.2 [20436464] with default parameters against the gene set in Ensembl Genomes release 14 (CADRE genebuild 3a). The sequencing data has been submitted to the European Nucleotide Archive (ENA) under accession ERP001382 (https://www.ebi.ac. uk/ena/data/view/ERP001382\&display=html).

\section{Data analysis}

The differentially regulated genes were analysed using FungiFun [60] to establish association with any functions or pathways in comparison with the non-differentially regulated genes. Gene enrichment analysis was carried out on the up-regulated and down-regulated genes, respectively, for FunCat [23] categories and the A. fumigatus annotated KEGG pathways [24]. A $p$ value cut-off of 0.05 was used.

\section{Real time PCR (qRT-PCR)}

RNA samples were DNase treated using a DNase kit supplied by Sigma-Aldrich. cDNA synthesis was performed using qScript ${ }^{\mathrm{TM}}$ cDNASuperMix (Quanta Biosciences) following the kit instructions. Primers used in this study are listed in Additional file 2: Table S14. The constitutively expressed gene, A. fumigatus calmodulin (calm) [61] was used as a reference gene. qRT-PCR was performed on the LightCycler ${ }^{\circ} 480$ Real-Time PCR System using the LightCycler $^{\circ} 1$ Sybr Green 1 Master Mix (Roche) as described previously [62]. qRT-PCR reactions for each gene were analysed in triplicate and were carried out for 40 cycles.

\section{Feeding experiments with $\left[{ }^{13} \mathrm{C}\right]$-phenylalanine}

A. fumigatus Af293 was cultured in Czapek-Dox Broth at $37^{\circ} \mathrm{C}$ with shaking at $200 \mathrm{rpm}$ in duplicate for $24 \mathrm{~h}$ before addition of gliotoxin $(5 \mu \mathrm{g} / \mathrm{ml}$ final) or methanol (solvent control) and $\left[{ }^{13} \mathrm{C}\right]$-phenylalanine $(10 \mu \mathrm{g} / \mathrm{ml}$ final) or water (solvent control). Cultures were incubated again at $37^{\circ} \mathrm{C}$ with shaking at $200 \mathrm{rpm}$. Culture supernatant was removed after $48 \mathrm{~h}$ and again after $72 \mathrm{~h}$. Supernatants were chloroform extracted, dried and resolubilised in methanol prior to LC-MS analysis as described previously [2].

\section{Whole proteome analysis}

A. fumigatus $\Delta$ gliT $T^{\mathrm{ATCC} 2633}$ was cultured in SabouraudDextrose media for $21 \mathrm{~h}$ followed by gliotoxin $(5 \mu \mathrm{g} / \mathrm{ml}$ final) or methanol addition for $3 \mathrm{~h}(n=4$ biological replicates for all specimens). Mycelial lysates were prepared in lysis buffer (100 mM Tris- $\mathrm{HCl}, 50 \mathrm{mM} \mathrm{NaCl}, 20 \mathrm{mM}$ EDTA, 10\% (v/v) Glycerol, $1 \mathrm{mM}$ PMSF, $1 \mu \mathrm{g} / \mathrm{ml}$ pepstatin $\mathrm{A}, \mathrm{pH}$ 7.5) with grinding, sonication and clarified using centrifugation. The resultant protein lysates were precipitated using TCA/acetone and resuspended in $8 \mathrm{M}$ Urea. After DTT reduction and IAA-mediated alkylation [63], sequencing grade trypsin combined with ProteaseMax surfactant was added. Digested samples were desalted prior to analysis using $\mathrm{C}_{18}$ spin columns (Thermo Scientific). All peptide mixtures were analysed via a Thermo Scientific Q-Exactive mass spectrometer coupled to a Dionex RSLCnano. LC gradients ran from 14-35\%B (A: 0.1\% (v/v) formic acid, B: $80 \%(\mathrm{v} / \mathrm{v})$ acetonitrile, $0.1 \%(\mathrm{v} / \mathrm{v})$ formic acid) over $2 \mathrm{~h}$, and data was collected using a Top15 method for MS/MS scans. Comparative proteome abundance and data analysis was performed using MaxQuant software (Version 1.3.0.5) [64], with Andromeda used for database searching and 
Perseus (Version 1.4.1.3) used to organise the data. Carbamidomethylation of cysteines was set as a fixed modification, while oxidation of methionines and acetylation of $\mathrm{N}$-termini were set as variable modifications. The maximum peptide/protein false discovery rates (FDR) were set to $1 \%$ based on comparison to a reverse database. The Label-Free Quantification (LFQ) algorithm was used to generate normalised spectral intensities and infer relative protein abundance. Proteins that matched to a contaminants database or the reverse database were removed and proteins were only retained in final analysis if detected in at least three replicates from at least one sample. Quantitative analysis was performed using a t-test to compare pairs of samples, and proteins with significant change in abundance ( $p$ value $<0.05$; fold change $\geq 2$ ) were included in the quantitative results. Qualitative analysis was also performed, to detect proteins that were found in at least 3 replicates of a particular sample, but undetectable in the comparitor sample.

\section{Secondary metabolite analysis}

A. fumigatus wild-type and $\Delta g l i T^{46645}$ were cultured in Czapeks-Dox broth for $96 \mathrm{~h}$ at $37^{\circ} \mathrm{C}$ at $200 \mathrm{rpm}$ in duplicate. Culture supernatants were organically extracted using an equal volume of chloroform and the extracts were dried by rota-evaporation and resuspended in methanol. Extracts were diluted $1 / 10$ in $0.01 \%$ formic acid prior to analysis by LC-MS as described previously [2].

\section{Cell viability analysis}

Following culturing and treatment as described above, mycelia were removed from the culture supernatant and washed with water. They were resuspended in $10 \mathrm{mM}$ HEPES before aliquots were plated on Sabouraud dextrose agar and incubated at $37^{\circ} \mathrm{C}$ for $24 \mathrm{~h}$. Viability was recorded.

\section{Additional files}

Additional file 1: Gene sequencing information. Combined FPKM values for all replicates, $\log _{2}$ fold changes and statistical significance data for all genes. Processed RNA-seq data. Relative expression of genes in $A$. fumigatus +/- gliotoxin; Relative expression of genes in A. fumigatus $\Delta$ gli $+/$ - gliotoxin

Additional file 2: Additional study information. Additional tables containing the functional categorisation of differentially regulated genes, Illumina RNA-seq summary statistics and primers used in the study. Additional Figure S1 is included. FunCat and KEGG categories of genes altered in expression; Summary Statistics for Illumina RNA-seq; Primers used for qRT-PCR; cell viability data.

\section{Abbreviations}

GSH: Glutathione; qRT-PCR: Quantitative real-time PCR; NRPS: Non-ribosomal peptide synthetase; PKS: Polyketide synthase; DTT: Dithiothreitol;

IAA: lodoacetamide.
Competing interests

The authors declare they have no competing interests.

\section{Authors' contributions}

GOK, SH, RAO, GWJ, and SD conceived and designed the experiments. SH and GOK extracted the RNA. TMK carried out library prep, sequencing and data mapping. Data analysis was performed by GOK, RAO, SH, TMK, DAF and SD. GOK and SH performed qPCR analysis. GOK carried out gliotoxin and secondary metabolite measurements and viability analysis. RAO performed the proteomics experimentation. GOK, GWJ and SD wrote the paper with contributions from RAO and TMK. All authors read and approved the final manuscript.

\section{Acknowledgements}

This work was supported by a Science Foundation Ireland Award to SD (SFI/ 11/PI/1188). SH was funded by the Irish Research Council. RAO was supported by $3 \cup$ Biomedical Research (DCU-NUI Maynooth-RCSI). qRT-PCR instrumentation was funded by Science Foundation Ireland (SFI/07/RFP/GEN/ F571/EC07). LFQ mass spectrometry facilities were funded by Science Foundation Ireland (12/RI/2346(3)). Agilent 6340 Ion Trap mass spectrometer was funded by the Higher Education Authority. We thank Prof. Nancy Keller ( $U$. Wisconsin-Madison) for provision of relevant mutant strains.

\section{Author details}

${ }^{1}$ Department of Biology, National University of Ireland Maynooth, Maynooth, Co. Kildare, Ireland. ${ }^{2}$ The Wellcome Trust Sanger Institute, Hinxton, Cambridge CB10 1SA, UK.

Received: 18 April 2014 Accepted: 7 October 2014

Published: 14 October 2014

\section{References}

1. Bernardo $\mathrm{PH}$, Brasch $\mathrm{N}$, Chai CLL, Waring P: A novel redox mechanism for the glutathione-dependent reversible uptake of a fungal toxin in cells. J Biol Chem 2003, 278:46549-46555.

2. Schrettl M, Carberry S, Kavanagh K, Haas H, Jones GW, Brien O, Nolan A, Stephens J, Fenelon O, Doyle S: Self-protection against Gliotoxin - a component of the Gliotoxin Biosynthetic Cluster, GliT, completely Protects Aspergillus fumigatus against Exogenous Gliotoxin. PLOS Pathog 2010, 6:e1000952. doi:10.1371/journal.ppat.1000952.

3. Gardiner DM, Howlett BJ: Bioinformatic and expression analysis of the putative gliotoxin biosynthetic gene cluster of Aspergillus fumigatus. FEMS Microbiol Lett 2005, 248:241-248.

4. Coleman JJ, Ghosh S, Okoli I, Mylonakis E: Antifungal activity of microbial secondary metabolites. PLoS One 2011, 6:e25321.

5. Carberry S, Molloy E, Hammel S, O'Keeffe G, Jones GW, Kavanagh K, Doyle S: Gliotoxin effects on fungal growth: mechanisms and exploitation. Fungal Genet Biol 2012, 49:302-312.

6. Gallagher L, Owens RA, Dolan SK, O'Keeffe G, Schrettl M, Kavanagh K, Jones GW, Doyle S: The Aspergillus fumigatus protein Glik protects against oxidative stress and is essential for gliotoxin biosynthesis. Eukaryot Cell 2012, 11:1226-1238.

7. Shim H, Sup J, Kim J, Won S, Kwon HJ: Discovery of gliotoxin as a new small molecule targeting thioredoxin redox system. Biochem Biophys Res Commun 2007, 359:523-528.

8. Gardiner DM, Cozijnsen AJ, Wilson LM, Pedras MSC, Howlett BJ: The sirodesmin biosynthetic gene cluster of the plant pathogenic fungus Leptosphaeria maculans. Mol Microbiol 2004, 53:1307-1318.

9. Cramer RA, Gamcsik MP, Brooking RM, Najvar LK, Kirkpatrick WR, Patterson TF, Balibar CJ, Graybill JR, Perfect JR, Abraham SN, Steinbach WJ: Disruption of a nonribosomal peptide synthetase in Aspergillus fumigatus eliminates gliotoxin production. Eukaryot Cell 2006, 5:972-980.

10. Bok JW, Chung D, Balajee SA, Marr KA, Andes D, Nielsen KF, Frisvad JC, Kirby KA, Keller NP: GliZ, a transcriptional regulator of Gliotoxin Biosynthesis, contributes to Aspergillus fumigatus Virulence. Infect Immun 2006, 74:6761-6768.

11. Forseth RR, Fox EM, Chung D, Howlett BJ, Keller NP, Schroeder FC: Identification of cryptic products of the gliotoxin gene cluster using NMR-based comparative metabolomics and a model for gliotoxin biosynthesis. J Am Chem Soc 2011, 133:9678-9681. 
12. Balibar CJ, Walsh CT: GliP, a multimodular nonribosomal peptide synthetase in Aspergillus fumigatus, makes the diketopiperazine scaffold of gliotoxin. Biochemistry 2006, 45:15029-15038.

13. Gardiner DM, Jarvis RS, Howlett BJ: The ABC transporter gene in the sirodesmin biosynthetic gene cluster of Leptosphaeria maculans is not essential for sirodesmin production but facilitates self-protection. Fungal Genet Biol 2005, 42:257-263.

14. Wang D, Toyotome T, Muraosa Y, Watanabe A, Wuren T, Bunsupa S, Aoyagi K, Yamazaki M, Takino M, Kamei K: GliA in Aspergillus fumigatus is required for its tolerance to gliotoxin and affects the amount of extracellular and intracellular gliotoxin. Med Mycol 2014, 52:506-518.

15. Scharf $D$, Remme $N$ : Transannular disulfide formation in gliotoxin biosynthesis and its role in self-resistance of the human pathogen Aspergillus fumigatus. J Am Chem Soc 2010, 132:10136-10141.

16. Qin Z, Baker AT, Raab A, Huang S, Wang T, Yu Y, Jaspars M, Secombes CJ, Deng $H$ : The fish pathogen Yersinia ruckeri produces holomycin and uses an RNA methyltransferase for self-resistance. J Biol Chem 2013, 288:14688-14697.

17. Li B, Walsh C: Streptomyces clavuligerus $\mathrm{HIml}$ is an intramolecular disulfide-forming dithiol oxidase in holomycin biosynthesis. Biochemistry 2011, 50:4615-4622

18. Gibbons JG, Beauvais A, Beau R, McGary KL, Latgé J-P, Rokas A: Global transcriptome changes underlying colony growth in the opportunistic human pathogen Aspergillus fumigatus. Eukaryot Cell 2012, 11:68-78.

19. Yu J, Fedorova ND, Montalbano BG, Bhatnagar D, Cleveland TE, Bennett JW, Nierman WC: Tight control of mycotoxin biosynthesis gene expression in Aspergillus flavus by temperature as revealed by RNA-Seq. FEMS Microbiol Lett 2011, 322:145-149.

20. Dhingra $S$, Andes D, Calvo AM: VeA regulates conidiation, gliotoxin production, and protease activity in the opportunistic human pathogen Aspergillus fumigatus. Eukaryot Cell 2012, 11:1531-1543.

21. Amich J, Schafferer $L$, Haas $H$, Krappmann S: Regulation of sulphur assimilation is essential for virulence and affects iron homeostasis of the human-pathogenic mould Aspergillus fumigatus. PLoS Pathog 2013, 9:e1003573.

22. Dhingra $\mathrm{S}$, Lind $\mathrm{AL}$, Lin H-C, Tang $Y$, Rokas A, Calvo AM: The fumagillin gene cluster, an example of hundreds of genes under veA control in Aspergillus fumigatus. PLoS One 2013, 8:e77147

23. Ruepp A, Zollner A, Maier D, Albermann K, Hani J, Mokrejs M, Tetko I, Güldener U, Mannhaupt G, Münsterkötter M, Mewes HW: The FunCat, a functional annotation scheme for systematic classification of proteins from whole genomes. Nucleic Acids Res 2004, 32:5539-5545.

24. Kanehisa M, Goto S: KEGG: kyoto encyclopedia of genes and genomes. Nucleic Acids Res 2000, 28:27-30.

25. Mitsuguchi H, Seshime Y, Fujii I, Shibuya M, Ebizuka Y, Kushiro T: Biosynthesis of steroidal antibiotic fusidanes: functional analysis of oxidosqualene cyclase and subsequent tailoring enzymes from Aspergillus fumigatus. J Am Chem Soc 2009, 131:6402-6411.

26. Perrin RM, Fedorova ND, Bok JW, Cramer RA, Wortman JR, Kim HS, Nierman WC, Keller NP: Transcriptional regulation of chemical diversity in Aspergillus fumigatus by LaeA. PLoS Pathog 2007, 3:e50.

27. Wiemann P, Guo C-J, Palmer JM, Sekonyela R, Wang CCC, Keller NP: Prototype of an intertwined secondary-metabolite supercluster. Proc Natl Acad Sci U S A 2013, 110:17065-17070.

28. Maiya S, Grundmann A, Li X, Li S-M, Turner G: Identification of a hybrid PKS/NRPS required for pseurotin A biosynthesis in the human pathogen Aspergillus fumigatus. Chembiochem 2007, 8:1736-1743.

29. Maiya S, Grundmann A, Li S-M, Turner G: The fumitremorgin gene cluster of Aspergillus fumigatus: identification of a gene encoding brevianamide F synthetase. Chembiochem 2006, 7:1062-1069.

30. Lin H-C, Chooi Y-H, Dhingra S, Xu W, Calvo AM, Tang Y: The fumagillin biosynthetic gene cluster in Aspergillus fumigatus encodes a cryptic terpene cyclase involved in the formation of $\beta$-trans-bergamotene. J Am Chem Soc 2013, 135:4616-4619.

31. Carberry S, Neville CM, Kavanagh KA, Doyle S: Analysis of major intracellular proteins of Aspergillus fumigatus by MALDI mass spectrometry: identification and characterisation of an elongation factor 1B protein with glutathione transferase activity. Biochem Biophys Res Commun 2006, 341:1096-1104.

32. Bok JW, Keller NP: LaeA, a Regulator of Secondary Metabolism in Aspergillus spp. Eukaryot Cell 2004, 3:527-535.
33. Lodeiro S, Xiong Q, Wilson WK, Ivanova Y, Smith ML, May GS, Matsuda SPT: Protostadienol biosynthesis and metabolism in the pathogenic fungus Aspergillus fumigatus. Org Lett 2009, 11:1241-1244.

34. Park H-S, Bayram O, Braus GH, Kim SC, Yu J-H: Characterization of the velvet regulators in Aspergillus fumigatus. Mol Microbiol 2012, 86:937-953.

35. Haas H: Iron - a key nexus in the Virulence of Aspergillus fumigatus. Front Microbiol 2012, 3:28.

36. Schrettl M, Bignell E, Kragl C, Sabiha Y: Distinct roles for intra-and extracellular siderophores during Aspergillus fumigatus infection. PLoS Pathog 2007 3:1195-1207.

37. Yasmin S, Alcazar-Fuoli L, Gründlinger M, Puempel T, Cairns T, Blatzer M, Lopez JF, Grimalt JO, Bignell E, Haas H: Mevalonate governs interdependency of ergosterol and siderophore biosyntheses in the fungal pathogen Aspergillus fumigatus. Proc Natl Acad Sci U S A 2012, 109:E497-E504.

38. Eisendle $M$, Oberegger $H$, Zadra I, Haas $H$ : The siderophore system is essential for viability of Aspergillus nidulans: functional analysis of two genes encoding l-ornithine N 5-monooxygenase (sidA) and a non-ribosomal peptide synthetase (sidC). Mol Microbiol 2003, 49:359-375

39. Arnaud MB, Chibucos MC, Costanzo MC, Crabtree J, Inglis DO, Lotia A, Orvis J, Shah P, Skrzypek MS, Binkley G, Miyasato SR, Wortman JR, Sherlock G: The Aspergillus Genome Database, a curated comparative genomics resource for gene, protein and sequence information for the Aspergillus research community. Nucleic Acids Res 2010, 38(Database issue):D420-D427.

40. Hartmann T, Cairns TC, Olbermann P, Morschhäuser J, Bignell EM, Krappmann S: Oligopeptide transport and regulation of extracellular proteolysis are required for growth of Aspergillus fumigatus on complex substrates but not for virulence. Mol Microbiol 2011, 82:917-935.

41. Bergmann A, Hartmann T, Cairns T, Bignell EM, Krappmann S: A regulator of Aspergillus fumigatus extracellular proteolytic activity is dispensable for virulence. Infect Immun 2009, 77:4041-4050.

42. Nishida $\mathrm{H}$ : Conservation of nucleosome positions in duplicated and orthologous gene pairs. ScientificWorldJournal 2012, 2012:298174.

43. Kupfahl C, Heinekamp T: The gliP gene of Aspergillus fumigatus results in loss of gliotoxin production but has no effect on virulence of the fungus in a low-dose mouse infection model. Mol Microbiol 2006, 62:292-302.

44. Sekonyela R, Palmer JM, Bok J-W, Jain S, Berthier E, Forseth R, Schroeder F, Keller NP: RsmA regulates Aspergillus fumigatus gliotoxin cluster metabolites including cyclo(L-Phe-L-Ser), a potential new diagnostic marker for invasive aspergillosis. PLoS One 2013, 8:e62591.

45. Li B, Forseth RR, Bowers AA, Schroeder FC, Walsh CT: A backup plan for self-protection: S-methylation of holomycin biosynthetic intermediates in Streptomyces clavuligerus. Chembiochem 2012, 13:2521-2526.

46. Dolan SK, Owens RA, O'Keeffe G, Hammel S, Fitzpatrick DA, Jones GW, Doyle S: Regulation of nonribosomal peptide synthesis: bis -thiomethylation attenuates gliotoxin biosynthesis in Aspergillus fumigatus. Chem Biol 2014, 21:999-1012.

47. Sanchez JF, Somoza AD, Keller NP, Wang CCC: Advances in Aspergillus secondary metabolite research in the post-genomic era. Nat Prod Rep 2012, 29:351-371.

48. Kato N, Suzuki H, Takagi H, Asami Y, Kakeya H, Uramoto M, Usui T, Takahashi $\mathrm{S}$, Sugimoto $\mathrm{Y}$, Osada $\mathrm{H}$ : Identification of cytochrome $\mathrm{P} 450$ s required for fumitremorgin biosynthesis in Aspergillus fumigatus. Chembiochem 2009, 10:920-928

49. Bok J, Balajee S, Marr K: LaeA, a regulator of morphogenetic fungal virulence factors. Eukaryot Cell 2005, 4:1574-1582.

50. Bayram O, Krappmann S, Ni M, Bok JW, Helmstaedt K, Valerius O, Braus-Stromeyer S, Kwon N-J, Keller NP, Yu J-H, Braus GH: VelB/NeA/LaeA complex coordinates light signal with fungal development and secondary metabolism. Science 2008, 320:1504-1506.

51. Wallner A, Blatzer M, Schrettl M, Sarg B, Lindner H, Haas H: Ferricrocin, a siderophore involved in intra- and transcellular iron distribution in Aspergillus fumigatus. Appl Environ Microbiol 2009, 75:4194-4196.

52. Xu XM, Møller SG: Iron-sulfur clusters: biogenesis, molecular mechanisms, and their functional significance. Antioxid Redox Signal 2011, 15:271-307.

53. Rutherford JC, Ojeda L, Balk J, Mühlenhoff U, Lill R, Winge DR: Activation of the iron regulon by the yeast Aft1/Aft2 transcription factors depends on mitochondrial but not cytosolic iron-sulfur protein biogenesis. J Biol Chem 2005, 280:10135-10140.

54. Sipos K, Lange H, Fekete Z, Ullmann P, Lill R, Kispal G: Maturation of cytosolic iron-sulfur proteins requires glutathione. J Biol Chem 2002, 277:26944-26949. 
55. Kumar C, lgbaria A, D'Autreaux B, Planson A-G, Junot C, Godat E, Bachhawat AK, Delaunay-Moisan A, Toledano MB: Glutathione revisited: a vital function in iron metabolism and ancillary role in thiol-redox control. EMBO J 2011, 30:2044-2056.

56. Schrettl M, Beckmann N, Varga J, Heinekamp T, Jacobsen ID, Jöchl C, Moussa TA, Wang S, Gsaller F, Blatzer M, Werner ER, Niermann WC, Brakhage AA, Haas H: HapX-mediated adaption to iron starvation is crucial for virulence of Aspergillus fumigatus. PLoS Pathog 2010, 6:e1001124.

57. Schrettl M, Kim HS, Eisendle M, Kragl C, Nierman WC, Heinekamp T, Werner ER, Jacobsen I, Illmer P, Yi H, Brakhage AA, Haas H: SreA-mediated iron regulation in Aspergillus fumigatus. Mol Microbiol 2008, 70:27-43.

58. Lee IR, Morrow CA, Fraser JA: Nitrogen regulation of virulence in clinically prevalent fungal pathogens. FEMS Microbiol Lett 2013, 345:77-84.

59. Wong KH, Hynes MJ, Davis MA: Recent advances in nitrogen regulation: a comparison between Saccharomyces cerevisiae and filamentous fungi. Eukaryot Cell 2008, 7:917-925.

60. Priebe S, Linde J, Albrecht D, Guthke R, Brakhage AA: FungiFun: A web-based application for functional categorization of fungal genes and proteins. Fungal Genet Biol 2011, 48:353-358.

61. Burns C, Geraghty R, Neville C, Murphy A, Kavanagh K, Doyle S: Identification, cloning, and functional expression of three glutathione transferase genes from Aspergillus fumigatus. Fungal Genet Biol 2005, 42:319-327.

62. O'Hanlon KA, Cairns T, Stack D, Schrettl M, Bignell EM, Kavanagh K, Miggin SM, O'Keeffe G, Larsen TO, Doyle S: Targeted disruption of nonribosomal peptide synthetase pes 3 augments the virulence of Aspergillus fumigatus. Infect Immun 2011, 79:3978-3992.

63. Collins C, Keane TM, Turner DJ, O'Keeffe G, Fitzpatrick DA, Doyle S: Genomic and proteomic dissection of the ubiquitous plant pathogen, Armillaria mellea: toward a new infection model system. J Proteome Res 2013, $12: 2552-2570$

64. Cox J, Mann M: MaxQuant enables high peptide identification rates, individualized p.p.b.-range mass accuracies and proteome-wide protein quantification. Nat Biotechnol 2008, 26:1367-1372.

doi:10.1186/1471-2164-15-894

Cite this article as: O'Keeffe et al:: RNA-seq reveals the pan-

transcriptomic impact of attenuating the gliotoxin self-protection mechanism in Aspergillus fumigatus. BMC Genomics 2014 15:894.

\section{Submit your next manuscript to BioMed Central and take full advantage of:}

- Convenient online submission

- Thorough peer review

- No space constraints or color figure charges

- Immediate publication on acceptance

- Inclusion in PubMed, CAS, Scopus and Google Scholar

- Research which is freely available for redistribution 